Int. J. Speleol. 10 (1978), pp. 73 - 105

\title{
Microflore et activité de groupements fonctionnels dans les sédiments de trois grottes de l'Italie Centrale
}

\author{
par
}

\author{
A. PASQUALINI, B. FUMANTI et L. VISONA் $\grave{(1)}^{(1)}$
}

Microflora and activity of functional groups in the sediments of three caves of Central Italy.

\section{SUMMARY}

A comparative study is presented of the microflora of sediments in three caves with different trophic characteristics.

Quantitative and qualitative variations of microflora were investigated for one year by studying the total microflora and the activity of the nitrogen cycle functional groups. Data were compared with those of other works of previous authors.

Results show that the quantity and kind of organic matter of sediment are most important factors regulating the abundance and activity of cavernicolous microflora.

The experimental part of this paper is prefaced by a digest of investigations previously carried out on cavernicolous microflora and by a report of topographic, geomorphologic, hydrologic and trophic characteristics of the caves examined.

\section{INTRODUCTION}

La présence d'une microflore autotrophe et hétérotrophe dans le milieu cavernicole fut mise en évidence pour la première fois en 1930 par E. Dudich. En particulier, la découverte de Bactéries autotrophes dans les grottes revêtait une importance considérable puisque ces Bactéries représentaient les seuls organismes cavernicoles capables de produire des composés organiques en utilisant des substrats minéraux; donc des organismes qui, du point de vue trophique, ne dépendent pas des apports organiques externes.

Aux travaux de Dudich $(1930,1932,1933)$ suivirent ceux de Magdeburg (1933, 1935) qui attribua les patines noirâtres qui recouvrent les parois de certaines grottes, aux métabolites de Bactéries nitrifiantes. Politi (1940) mit ensuite en rapport les phénomènes de noircissement des concrétions des grottes de «Po-

\footnotetext{
${ }^{(1)}$ Istituto Botanico, Università di Roma.

Città Universitaria. Roma. Italia.
} 
stojna» (Istrie) avec la présence de microorganismes. Successivement Lovett (1949) en Grande-Bretagne, Birstein et Borutzky (1950) en URSS et Liddo (1951) dans les grottes de «Castellana» (Italie), mirent en évidence la présence de microorganismes dans les différents milieux cavernicoles.

A partir de 1957, avec les recherches de Caumartin (1957a, 1957b, 1959a, 1959b, 1960, 1961, 1962, 1963, 1964a, 1964b, 1964c, 1965), la microbiologie des grottes a acquis un rôle important dans la spéléobiologie. Caumartin a isolé une Bactérie constamment présente dans les grottes, le Perabacterium spelei, Ferrobactérie autotrophe et microaérobie capable de fixer l'azote gazeux et de mener, dans des conditions déterminées, une vie hétérotrophe. Par la suite, Caumartin et Renault (1958) ont mis en évidence l'intense activité des microorganismes, et plus particulièrement des Ferrobactéries, sur les concrétions, sur les roches, dans les argiles des grottes, et ont formulé une hypothèse biochimique sur l'évolution des concrétions et sur la genèse du mondmilch, attribuant un rôle fondamental à la présence du Perabacterium spelei et d'autres Bactéries capables de désagréger le substrat. Du mondmilch se sont successivement occupés Mason-Williams (1959), Pochon et coll. (1964), Caumartin (1964c) et Bertouille (1972), ce dernier a observé une nitrification active due aux Bactéries qui appartiennent au genre «Nitrosomonas» et au genre «Nitrobacter».

Les recherches de Mason-Williams et Benson-Evans (1958) en GrandeBretagne, de Fischer (1959a, 1959b) et de Chodorowski (1959) en Pologne, de Varga et Takats (1960) en Hongrie, de Martini (1962a, 1962b, 1962c, 1963) en Italie, ont mis en évidence, dans les grottes, la présence de microorganismes capables d'effectuer les différentes activités fonctionnelles du cycle de l'azote, du soufre, du carbone et du phosphore.

D'autres auteurs se sont occupés des microorganismes des différents milieux cavernicoles et en ont évalué les aspects quantitatifs et qualitatifs: Molnar (1961), Luppi-Mosca et Campanino (1962), Orpurt (1964), Bene et coll. (1964), Pohl et White (1965), Mason-Williams (1965), Perrier (1970, 1971), Barr et Kuehne (1971).

L'importance de la microflore des sédiments dans le trophisme des animaux cavernicoles a été mise en relief par Ginet (1960) et par Gounot (1960) qui ont étudié le rôle du limon dans l'alimentation du "Niphargus». Gounot (1964, 1966, 1967, 1969a, 1969b) a en outre évalué l'activité productrice de la microflore des limons de grotte à travers l'étude des activités fonctionnelles et du pouvoir de synthèse des facteurs de croissance. Gounot $(1968,1969 \mathrm{c}, 1970 \mathrm{a}$, 1970b, 1973, 1974) a étudié aussi les effets sélectifs de température sur les microorganismes des grottes.

\section{DESCRIPTION DES GROTTES}

Les grottes choisies pour nos recherches se trouvent dans trois différentes zones karstiques de l'Italie Centrale (fig. 1). Il s'agit de la grotte de «Punta degli 


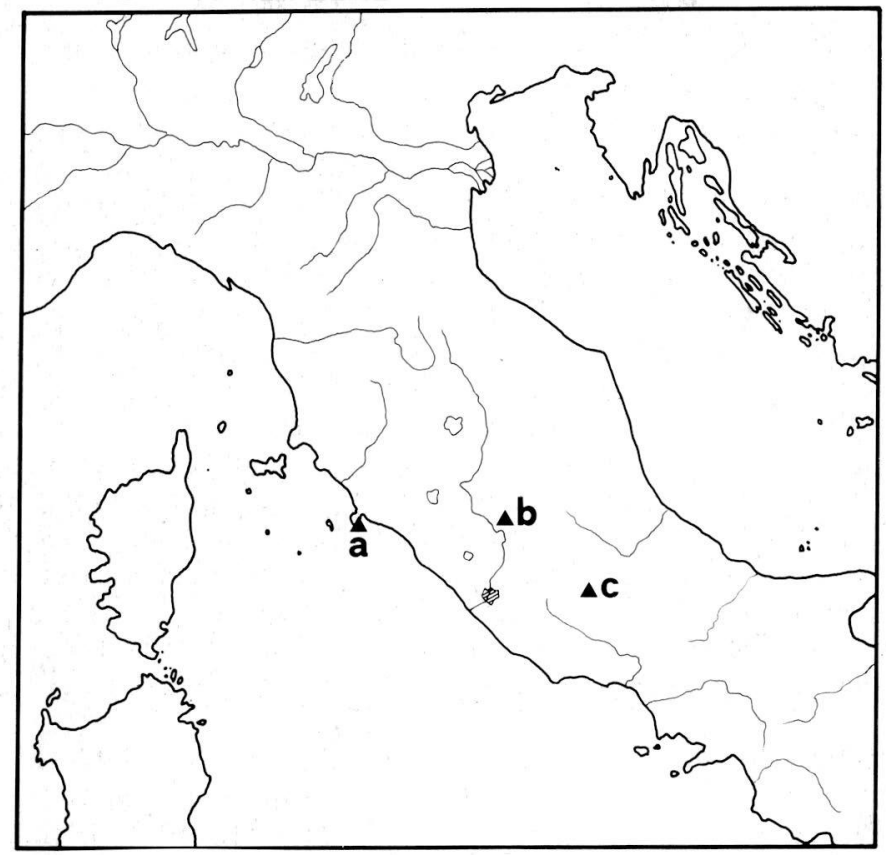

Fig. 1. - Localisation des grottes étudies:

a) Grotte de «Punta degli Stretti»

b) Grotte de la «Mandorla»

c) Résurgence de «Trevi»

Stretti» dans le Mont Argentario, de la grotte de la «Mandorla» dans les montagnes de la Sabina du Nord, de la résurgence de «Trevi» dans les Monts Ernici.

Les trois cavités se trouvent à l'intérieur d'une bande géographique comprise entre $42^{\circ} 25^{\prime} 54^{\prime \prime}$ et $41^{\circ} 51^{\prime} 18^{\prime \prime}$ de latitude Nord. La position géographique, comme l'altitude moyenne, déterminent dans les trois grottes une condition climatique typiquement tempérée. Au contraire, du pont de vue trophique, la grotte de la «Mandorla» est une cavité distrophique, selon la classification de Sbordoni et coll. (1977), tandis que les deux autres sont oligotrophiques.

\section{GROTTE DE «PUNTA DEGLI STRETTI» Données générales}

Province: Grosseto. - Commune: Porto S. Stefano. - Localité: Punta degli Stretti. - Carte I.G.M.: $\mathrm{F}^{\circ} 135$ III N.E. - Longitude: $1^{\circ} 16^{\prime} 54^{\prime \prime} \mathrm{O}$. du méridien de Rome. - Latitude: $42^{\circ} 25^{\prime} 54^{\prime \prime}$ N. - Altitude: 15 m au-dessus de la mer. - Développement total: $1100 \mathrm{~m}$ - Bibliographie: Datti (1930), Ducci et Segre (1950), De Sio et Chiancone (1965). 
Caractéristiques topographiques, géomorphologiques et hydrologiques (fig. 2).

La grotte de «Punta degli Stretti» se trouve sur le versant Nord du Mont Argentario, relief qui forme le promontoire du même nom, uni à la Maremma toscane par deux cordons sablonneux qui renferment la lagune d'Orbetello. Le Monte Argentario est constitué surtout de calcaires triasiques en partie métamorphosés et minéralisés (Cumin, 1928; T.C.I., 1957).

La cavité s'ouvre près de la route qui mène à $\mathrm{S}$. Stefano, à $20 \mathrm{~m}$ de la lagune, avec une entrée petite et riche en végétation. A environ $25 \mathrm{~m}$ de cette entrée naturelle, un tunnel ferroviaire, désormais inutilisé, a coupé la grotte, ouvrant ainsi une petite entrée artificielle.

La grotte de «Punta degli Stretti» est une cavité «à stalactites», d'un développement horizontal d'environ un kilomètre. Très abondantes y sont des concrétions, avec de massives coulées stalactitiques et stalagmitiques et de nombreuses colonnes. Au contraire les sédiments ne sont pas abondants.

La couverture rocheuse est réduite, cela influence le régime des suintements qui présente sa phase maximum en automne et au printemps (la pluviosité de la région varie de 600 à $800 \mathrm{~mm}$ par an). La cavité présente diverses flaques et deux lacs alimentés par les suintements et par des sources souterraines. L'eau est douce, avec un $\mathrm{pH} 7$, malgré la proximité de la lagune.

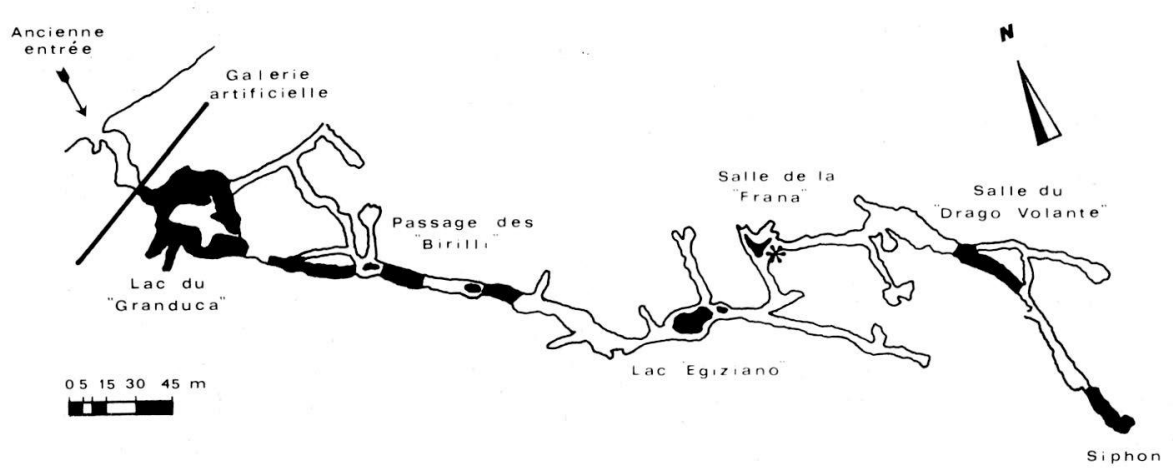

Fig. 2. - Grotte de «Punta degli Stretti»: Plan. ("point des prélèvements). 


\section{Apports trophiques.}

La grotte de «Punta degli Stretti» est une grotte tempérée typique, avec apports exogènes réduits et faune rare. Des Chauves-souris peuplent la première partie de la cavité où se trouvent des dépôts de guano. La couverture rocheuse réduite permet la pénétration, à travers les eaux d'infiltration, de débris végétaux plus ou moins décomposés. Très étudiée la faune des eaux (Stella, 1951a, 1951b, 1953, 1955, 1959; Baschieri-Salvadori, 1952; Stella et BaschieriSalvadori, 1953); où ont été trouvé des Crustacés troglobies de grand intérêt. De la faune terrestre ont été étudiés les Orthoptères (Lanza, 1954).

\section{Provenance des échantillons.}

Les échantillons de sédiment ont été prélevés dans la salle appelée «Sala della Frana» (fig. 2), à environ $320 \mathrm{~m}$ de l'entrée artificielle. La couverture rocheuse y est limitée, comme le démontre la présence de quelques fines racines. Le guano y est rare puisque les Chauves-souris peuplent d'autres zones de la grotte. Les échantillons ont été collectés dans une anfractuosité de la salle, à l'abri des courants d'air et du passage des Chauves-souris et d'éventuels visiteurs. Les sédiments sont partiellement recouverts d'une couche stalagmitique inactive qui les protège de pollutions possibles et que l'on a dû enlever en partie pour effectuer les prélèvements.

\section{GROTTE DE LA «MANDORLA»}

Données générales.

Province: Rieti - Comune: Contigliano - Localité: Colle Palaini - Carte I.G.M.: $\mathrm{F}^{\circ} 138$ II S.O. Longitude: $0^{\circ} 17^{\prime} 43^{\prime \prime}$ E. du méridien de Rome. - Latitude: $42^{\circ} 22^{\prime} 00^{\prime \prime}$ N. - Altitude: $640 \mathrm{~m}$ audessus de la mer - Développement total: $52 \mathrm{~m}$ - Dénivellation: + $5 \mathrm{~m}$ - Bibliographie: Riccardi (1927), Segre (1948).

Caractéristiques topographiques, géomorphologiques et hydrologiques (fig. 3).

La cavité se trouve dans les montagnes de la Sabina du Nord qui représentent géologiquement l'extrémité méridionale de l'Appennin de l'Ombrie-Marches. Les monts de la Sabina, constitués en grande partie de calcaires crétacés et liasiques, présentent des phénomènes karstiques isolés et modestes, limités en général au Lias, avec des grottes de petites dimensions. La pluviosité de la région varie entre 800 et $1000 \mathrm{~mm}$ par an, mais augmente avec l'altitude qui, toutefois, n'atteint pas de niveaux appréciables (Segre, 1948; Maxia, 1956).

La grotte de la "Mandorla» s'ouvre dans le calcaire du Crétacé inférieur, à $640 \mathrm{~m}$ d'altitude, à $6 \mathrm{~m}$ au-dessus du lit d'un torrent à sec, ce qui fait penser qu'il s'agit d'une résurgence fossile. La cavité a une couverture rocheuse très 
réduite, surmontée d'une épaisse végétation dont les racines (certaines de grandes dimensions) pénètrent à l'intérieur de la cavité. La grotte suit une diaclase, elle a une entrée de $5,50 \times 1,40 \mathrm{~m}$ et un développement légèrement ascendant, sur un total de $52 \mathrm{~m}$. La voûte est basse et la largeur est également réduite. Les eaux courantes sont absentes. Les suintements sont abondants en hiver et inexistants en été. Fréquentes sont les concrétions inactives.

\section{Apports trophiques.}

La «Mandorla» est une grotte climatiquement tempérée, mais avec des caractéristiques tropicales du point de vue trophique, avec grande abondance de racines, débris végétaux et avec du guano épars. La faune est riche et se compose d'Isopodes, Araignées, Chilopodes, Collemboles, Diplopodes, Orthoptères, Coléoptères, petits Mammifères. Dans les dix premiers mètres de la grotte, on trouve des patines d'Algues recouvrant la roche et, plus loin, de fréquentes moisissures qui se développent sur les débris organiques.

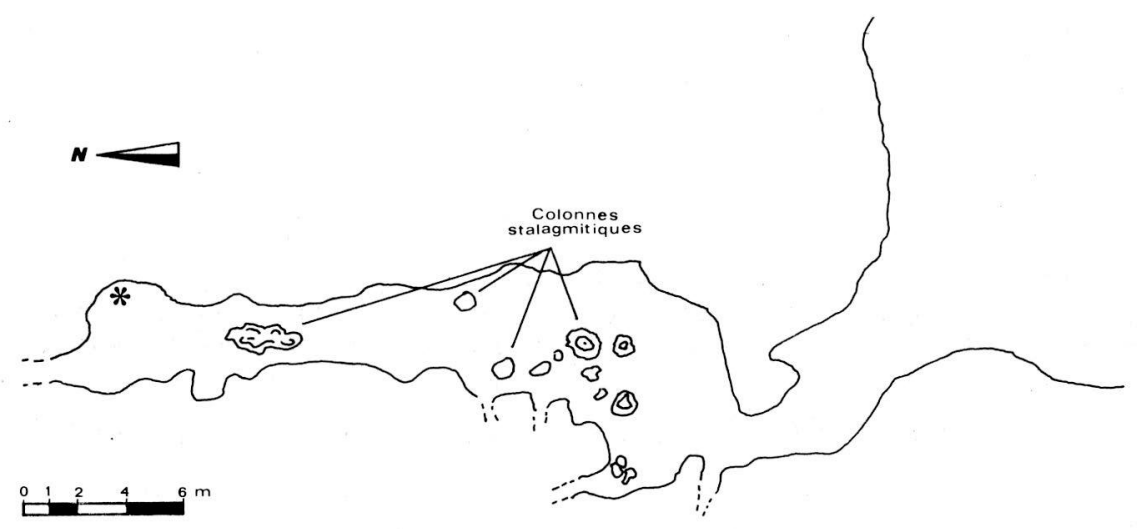

Fig. 3. - Grotte de la «Mandorla»: Plan. ("point des prélèvements). 


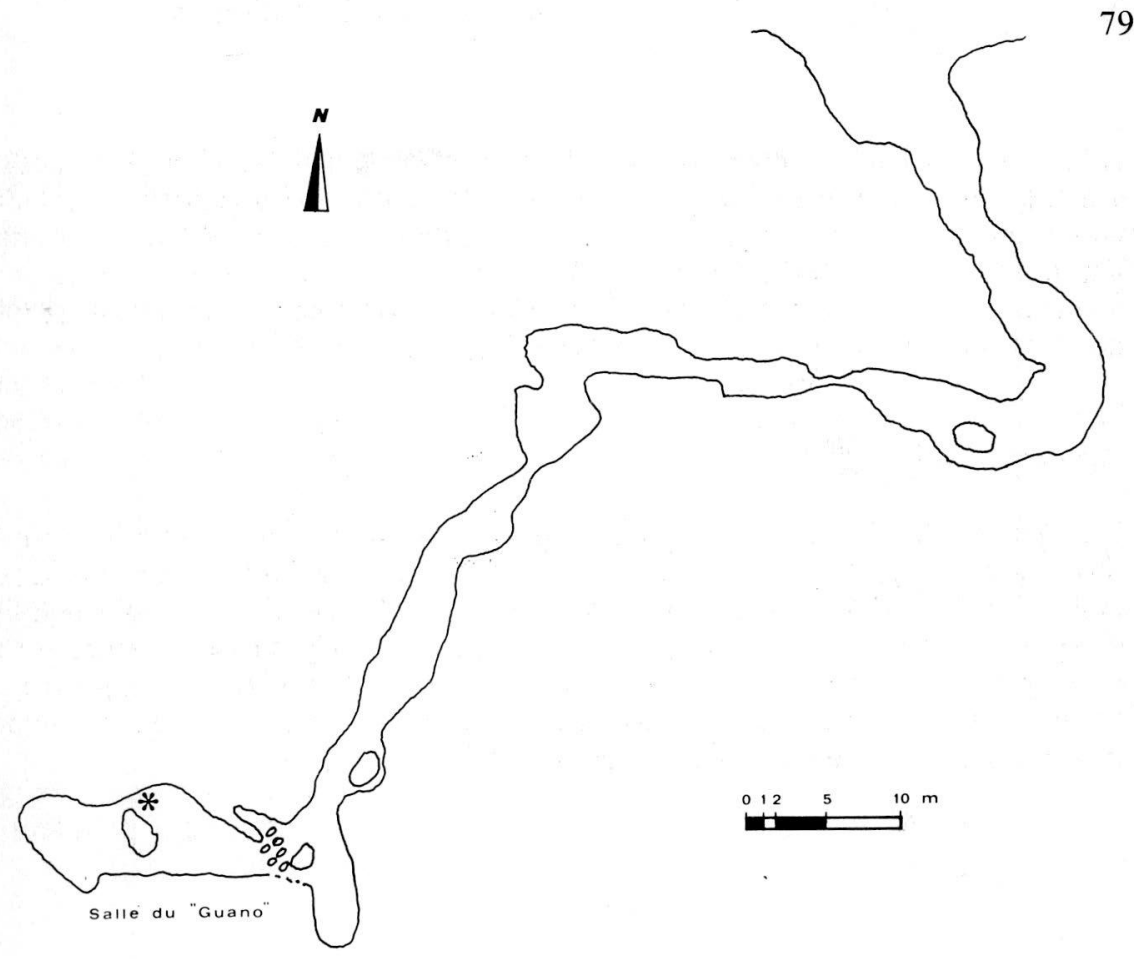

Fig. 4. - Résurgence de «Trevi»: Plan. (" point des prélèvements).

Provenance des échantillons.

Les prélèvements ont été effectués dans la salle terminale (fig. 3), petite et bas$\mathrm{se}$, à environ $40 \mathrm{~m}$ de l'entrée. La voûte est séparée de l'extérieur par une couche rocheuse limitée de laquelle pendent de fines racines. Des Chauves-souris peuplent cette petite salle. Le substrat est constitué d'une terre riche en débris végétaux et même animaux.

\section{RÉSURGENCE DE «TREVI» Données générales.}

Province: Frosinone - Commune: Trevi nel Lazio- Localité: sous les «Coste di Arcinazzo», près de la rivière Aniene - Carte I.G.M.: F ${ }^{\circ} 151$ I S.O. - Longitude: $0^{\circ} 45^{\prime} 35^{\prime}$ ' E. du méridien de Rome - Latitude: $41^{\circ} 51$ '18' N. - Altitude: $600 \mathrm{~m}$ au-dessus de la mer - Développement total: $119 \mathrm{~m}$ - Dénivellation: +13 m - Bibliographie: Boll. Ass. Spel. Rom. (sous presse).

Caractéristiques topographiques, géomorphologiques et hydrologiques (fig. 4)

La cavité se trouve dans les Monts «Ernici», au bord des plateaux d'Arcinaz- 
zo. Les reliefs sont formés de calcaires et de dolomies en grande partie datant du Crétacé. Le phénomène karstique y est très intense. La pluviosité y est forte, elle varie de 1200 à $1500 \mathrm{~mm}$ par an et augmente avec l'altitude qui atteint des hauteurs remarquables (Segre, 1948).

La résurgence de «Trevi» s'ouvre dans le calcaire crétacé à une altitude de 600 $\mathrm{m}$, aux alentours de la rivière Aniene. Il s'agit d'une modeste résurgence active, avec une entrée large et un développement ascendant, surtout dans la partie finale. Le développement total est de $119 \mathrm{~m}$ et la largeur maximum s'observe dans la salle du guano avec $6,5 \mathrm{~m}$. Abondantes sont les concrétions actives et l'argile.

Le petit ruisseau qui traverse presque toute la grotte se jette dans l'Aniene. Sa température est de $9,7^{\circ} \mathrm{C}$ (mai).

Durant la saison sèche, il n'en reste que quelques flaques. Les suintements sont abondants.

Apports trophiques.

La résurgence de «Trevi» est une grotte tempérée typique; les apports exogènes sont réduits et la faune rare. De nombreuses Chauves-souris peuplent la salle du guano où se trouve une abondante couche de guano.

\section{Provenance des échantillons.}

Les prélèvements ont été effectués sur l'argile qui recouvre les parois de la salle du guano (fig. 4), à plus de $100 \mathrm{~m}$ de l'entrée. Il s'agit d'un substrat très humide et dépourvu de guano.

\section{CARACTÉRISTIQUES PHYSICO-CHIMIQUES DES SÉDIMENTS EXAMINÉS}

Dans les sediments des trois grottes on a relevé les paramètres physicochimiques suivantes: temperature, teneur en eau, $\mathrm{pH}$, carbone organique, matière organique totale, azote total, rapport $\mathrm{C} / \mathrm{N}$, carbonates, granulométrie. Les résultats obtenus sont rapportés aux tableaux 1, 2, 3 .

\section{TEMPÉRATURE}

Les températures ont été mesurées avec un thermomètre au dixième de degré et se réfèrent à la superficie de contact terre/air des zones où ont été effectués les prélèvements.

Les valeurs obtenues, reportées au tableau 1, rentrent dans les limites caractéristiques des cavités de l'Italie Centrale, qui varient en général entre 7 et $12^{\circ} \mathrm{C}$ 


\section{$\begin{array}{lllll}\text { XI-72 } & \text { II-73 } & \text { V-73 } & \text { VII-73 } & \text { XI-73 }\end{array}$}

A) «Punta degli Stretti»

Extérieur

Entrée

Point des prélèvements

B) «Mandorla»

Extérieur

Entrée

Point des prélèvements

C) «Trevi»

Extérieur

Entrée

Point des prélèvements

$\begin{array}{llllc}16 & 9,5 & 20 & 26 & 16 \\ 14 & 10 & 16 & 16 & 14,5 \\ 15 & 15 & 15 & 16 & 16\end{array}$

$\begin{array}{ccccc}8 & 1,5 & 20 & 23,5 & 14 \\ 9 & 4 & 13 & 17 & 11,5 \\ 14,5 & 12 & 11 & 13 & 14,5\end{array}$

$\begin{array}{ccccc}3 & 1,5 & 20 & 23 & 6 \\ 7,5 & 3 & 15 & 19 & 10 \\ 9,5 & 9,5 & 9,5 & 9,5 & 10\end{array}$

Tableau 1 - Températures en ${ }^{\circ} \mathrm{C}$.

dans les grottes au-dessus de 500-600 m d'altitude et entre 12 et $18^{\circ} \mathrm{C}$ dans celles situées à une altitude inférieure (Segre, 1948).

Des trois grottes étudiées, celle de «Punta degli Stretti» est la plus chaude mais c'est également celle qui se trouve à l'altitude la plus basse, tandis que la résurgence de «Trevi» qui se situe à $600 \mathrm{~m}$ est la plus froide. Les températures de la grotte de la «Mandorla» sont plutôt élevées si l'on considère son altitude (640 $\mathrm{m})$.

Un indice important de la dépendance climatique des grottes par rapport à l'extérieur est représenté par l'amplitude thermique. La différence entre la température maximale et minimale est très réduite à «Trevi» $\left(0,5^{\circ} \mathrm{C}\right)$ et à «Punta degli Stretti» $\left(1^{\circ} \mathrm{C}\right)$, tandis qu'elle est plus importante à la «Mandorla» $\left(3,5^{\circ} \mathrm{C}\right)$. Le fait que l'amplitude thermique de la grotte de la «Mandorla» soit plus importante dépend de sa longueur réduite, de la mince couverture rocheuse et du type de circulation d'air de la cavité. La «Mandorla» est en effet un typique «cul de sac ascendant», c'est-à-dire qu'elle est caractérisée par une stagnation de l'air chaud en hiver et par une condition dynamique en été, quand l'air froid sort de la grotte au niveau du sol.

\section{TENEUR EN EAU}

La teneur en eau des échantillons a été évaluée en desséchant à $100^{\circ} \mathrm{C}$, pendant $24 \mathrm{~h}$, une certaine quantité de sédiment frais. De la différence entre le poids de l'échantillon frais et celui du même échantillon séché, on a obtenu le 
poids de l'échantillon frais et celui du même échantillon séché, on a obtenu le pourcentage d'eau présente.

Comme cela était à prévoir, les valeurs obtenues (tab. 2) étaient assez élevées et cela à cause de l'importante humidité typique du milieu cavernicole. Les sédiments de la grotte de «Punta degli Stretti» sont les moins humides des trois et présentent des valeurs assez stables, variant entre $21,7 \%$ et $32,5 \%$. Dans la résurgence de «Trevi» les variations sont plus sensibles: de 33,1\% à 49,6\%. C'est dans les sédiments de la grotte de la «Mandorla» que les variations sont les plus marquées: de $19,3 \%$ à $62,0 \%$, cela s'explique en grande partie par la finesse de la couverture rocheuse de cette grotte qui est ainsi plus sensible aux variations de pluviosité.

Les valeurs obtenues sont semblables à celles signalées par d'autres auteurs: Martini (1962a) a trouvé des teneurs en eau allant de 26,7\% à 33,0\% dans le terrain d'une grotte de l'Ombrie (Italie); Luppi-Mosca et Campanino (1962), dans les sédiments de deux grottes du Piémont (Italie), ont rélevé 20,85\% et $27,87 \%$ d'eau; Gounot (1970b), dans le limon de grottes norvégiennes a trouvé des valeurs d'humidité variant de $14,6 \%$ à $22,4 \%$.

$\mathrm{pH}$

Le pH des sédiments des trois grottes (tab. 3) est de 7,5 - 7,6. Cette alcalinité est normale dans le milieu cavernicole où la grande quantité de $\mathrm{Ca}^{++}$neutralise les acides des sédiments.

D'autres auteurs ont eux-aussi observé des valeurs analogues: Bögli (1961) 7,7 - 8,5; Martini (1962a), dans une grotte de l'Ombrie, 8,6 - 9,0; Luppi-Mosca et Campanino (1962), dans deux grottes piémontaises, 8,5 - 8,9; Orpurt (1964), dans une grotte des Bahamas, 7,1 - 9,9; Gounot, dans des grottes françaises (1967), 7,2 - 8,3 et, dans des grottes norvégiennes (1970b), 8,4 - 8,5. Seuls Varga et Takats (1960), dans la grotte «Baradla» (Hongrie), ont trouvé un pH acide de 5,3.

\section{CARBONE ORGANIQUE ET MATIÈRE ORGANIQUE TOTALE}

Pour le dosage du carbone organique on a suivi la méthode de Walkey-Black, basée sur l'oxydation par le bichromate de potassium et titrage par une solution de sel de Mohr. La matière organique totale été calculée en multipliant le pourcentage de carbone organique par la valeur standard de 1,726.

Ler résultats obtenus sont exprimés en pourcentage par rapport au poids de sédiment sec (tab. 3). Dans la grotte de «Punta degli Stretti» et dans la résurgence de «Trevi» les teneurs en matière organique n'atteignent pas $1 \%$. Il s'agit de pourcentages décidément bas, même par rapport à ceux signalés par différents auteurs dans les grottes des régions tempérées (tab. 4) qui varient d'un minimum de $0,026 \%$ à un maximum de $4,8 \%$ pour le carbone organique et d'un minimum de $1,48 \%$ à un maximum de $9,6 \%$ pour la matière organique 


\begin{tabular}{|c|c|}
\hline & $\begin{array}{l}\infty \\
\stackrel{\sim}{\sim} \\
=\end{array}$ \\
\hline & 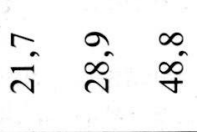 \\
\hline$\frac{1}{i}$ & 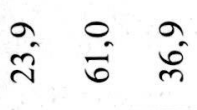 \\
\hline$\stackrel{f}{t}$ & فे \\
\hline & 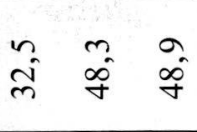 \\
\hline & 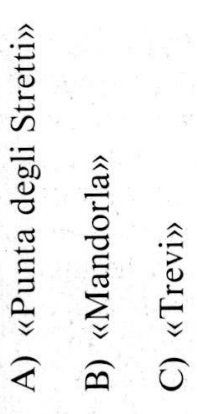 \\
\hline
\end{tabular}

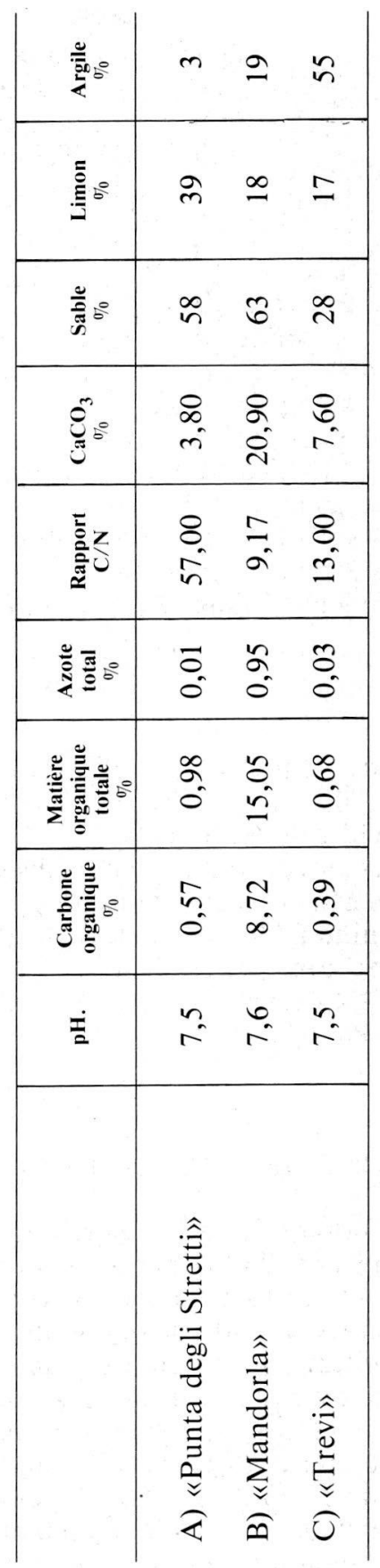


totale. Les taux de la grotte de la «Mandorla» sont au contraire très élevés: $8,72 \%$ de carbone organique et $15,05 \%$ de matière organique représentent des valeurs remarquables, même pour des sols de surface où la quantité de matière organique totale est en général comprise entre 1\% et 6\% (Lotti, 1956; Bardin, 1970). Ce pourcentage augmente dans les terrains organiques acides (tourbes) où il dépasse $10 \%$ et arrive même à 48\% (Lotti, 1956; De Barjac, 1956). Dans les sédiments boueux fluviaux, marins et des estuaires, les teneurs en matière organique totale vont de $2 \%$ à $33 \%$, atteignant un maximum de $90 \%$ lorsqu'il s'agit de certains sédiments lacustres (Boucart et Francis-Boeuf, 1942). Quant au carbone organique, des taux assez semblables à ceux des sédiments cavernicoles ont été signalés dans les limons des glaciers où Moiroud (1970) a trouvé une teneur d'à peine $0,3 \%$ en carbone organique par rapport au poids sec.

\section{AZOTE TOTAL}

Le dosage de l'azote total a été réalisé par la méthode de Kjeldahl, en oxydant l'azote organique en sulfate d'ammonium par traitement à chaud avec acide phospho-sulfurique. L'azote total se révèle être composé essentiellement d'azote organique.

Les résultats obtenus (tab. 3) sont exprimés en pourcentage par rapport au poids de sédiment sec. Le taux de $0,01 \%$ de la grotte de «Punta degli Stretti» et celui de $0,03 \%$ de la résurgence de «Trevi» sont des valeurs plutôt basses, même par rapport à celles relevées dans les sédiments d'autres grottes (tab. 4) où l'azote organique varie de $0,007 \%$ à $0,161 \%$.

Le taux de $0,95 \%$ d'azote total de la grotte de la «Mandorla» est très élevé, même par rapport aux sols de surface.

Dans les terrains de l'Agro Romano et des «Marcite» de la Lombardie (Italie), Schreiber $(1929,1930)$ a, en effet, signalé respectivement: 0,167 - 0,252 et $0,148-0,180 \%$ d'azote organique. Dans des terrains alcalins inorganiques, les taux sont en moyenne compris entre $0,1 \%$ et $0,3 \%$ (Lotti, 1956; Bardin, 1970), tandis que les terrains organique acides (tourbes) ils vont généralement de $0,2 \%$ à $2 \%$ (De Barjac, 1956; Lotti, 1956). Enfin, dans les sédiments des estuaires et encore plus dans les sédiments lacustres, on a trouvé des taux beaucoup plus élevés d'azote organique, jusqu'à plus de 3\% (Boucart et Francis-Boeuf, 1942; Stangenberg, 1949). Les sédiments pauvres en azote sont au contraire les limons des glaciers avec un taux de $0,01 \%$ d'azote total (Moiroud, 1970).

\section{RAPPORT C/N}

Le rapport carbone organique total/azote total (Pochon et De Barjac, 1958) est un indice important de l'activité microbienne du terrain et permet l'évaluation du type prédominat d'apport organique. Les valeurs obtenues (tab. 3) à la résurgence de «Trevi» et à la grotte de la «Mandorla» sont assez semblables à 


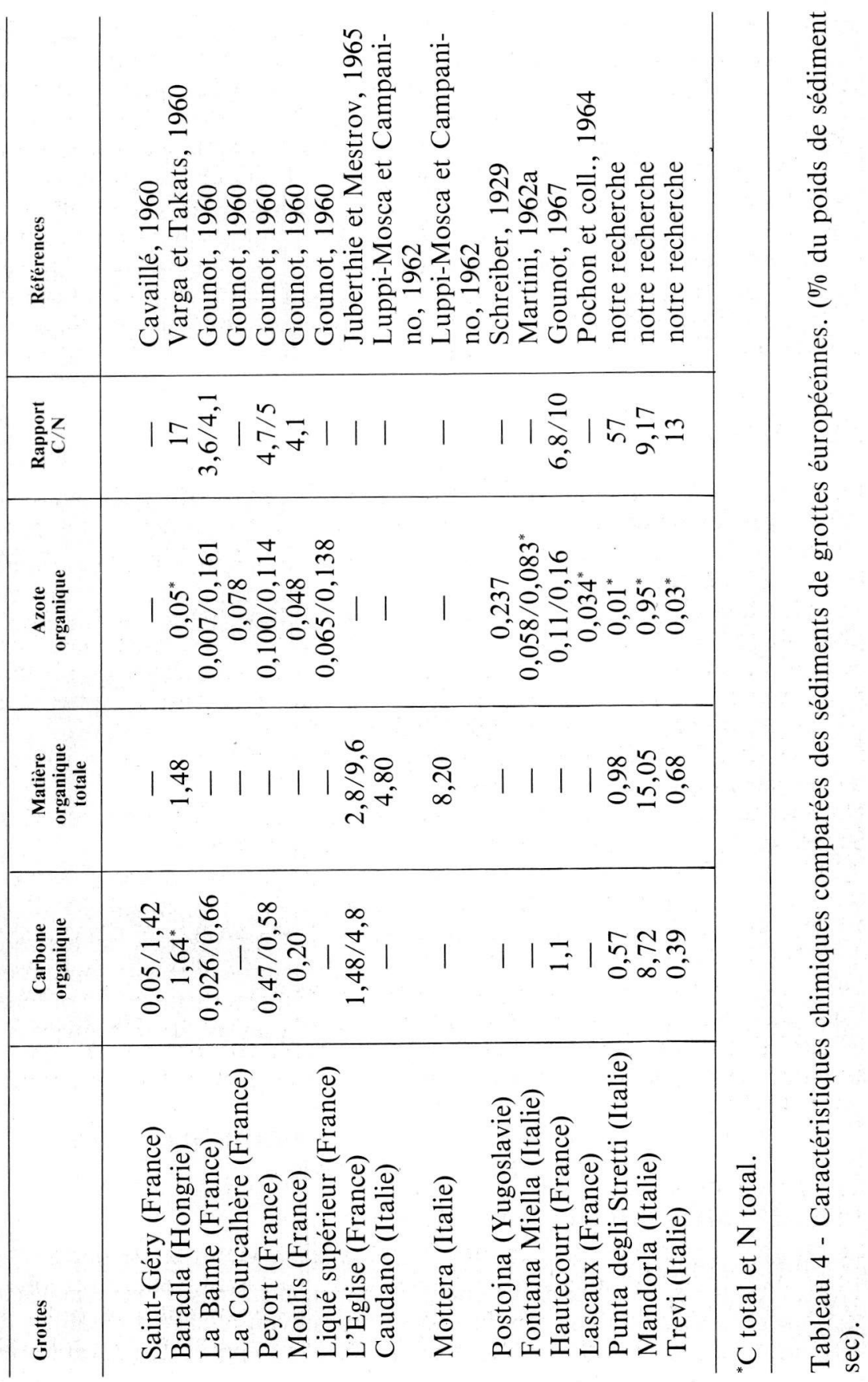


celles relevées dans d'autres grottes européennes (tab. 4). Au contraire, le rapport $\mathrm{C} / \mathrm{N}$ de la grotte de «Punta degli Stretti», qui est de 57, se détache fortement de la moyenne et indique la pénétration de substances d'origine essentiellement végétale.

Le rapport $\mathrm{C} / \mathrm{N}$ dans les sédiments lacustres, fluviaux et marins varie entre 10 et 15 (Boucart et Francis-Boeuf, 1942); De Barjac (1956) a trouvé, en tourbe acide, des valeurs de $\mathrm{C} / \mathrm{N}$ comprises entre 11,6 et 18,5 et Bardin (1970) des valeurs comprises entre 7,8 et 11,7 en sols alcalins d'une vallée alpine.

Pochon et De Barjac (1958) supposent que l'activité microbienne su sol, en produisant des protéines et des substances azotées, est une cause de l'abaissement du rapport C/N. Cette observation a été appliquée par Gounot (1960, 1967) aux limons argileux de grotte.

\section{CARBONATES}

Pour la détermination des carbonates on a utilisé la méthode gazvolumétrique, au moyen d'un calcimètre. Dans les sédiments des trois grottes la teneur en carbonates, constitués essentiellement de $\mathrm{CaCO}_{3}$, est comprise entre $3,80 \%$ et $20,90 \%$ du poids de sédiment sec (tab. 3). Il s'agit de taux élevés pour la résurgence de «Trevi» et surtout pour la grotte de la «Mandorla», qui s'expliquent par la géologie calcaire des grottes.

Gounot (1960) dans le limon des gours de différentes grottes françaises a trouvé des taux en carbonates compris entre $3,9 \%$ et $74 \%$ du poids de limon sec. Bögli (1961) a obtenu des valeurs de 12,3 - 50,1\% et Martini (1962a) de 65 $-70 \%$.

\section{GRANULOMÉTRIE}

Pour l'analyse granulométrique des sédiments on a suivi la méthode densimétrique, en utilisant l'hydromètre de Bouyoucos. Les résultats obtenus sont reportés au tableau 3. Ils indiquent des sédiments moyennement sablonneux à «Punta degli Stretti» et à la «Mandorla», tandis que les valeurs granulométriques de la résurgence de «Trevi» correspondent à un sédiment argileux.

\section{TECHNIQUES DE L'ANALYSE MICROBIOLOGIQUE DES SÉDIMENTS}

\section{PRÉLÈVEMENT DES ÉCHANTILLONS}

Les prélèvements ont toujours été effectués au même endroit dans les trois grottes, dans des zones complètement obscures, exemptes d'évenctuelles inondations et loin des entrées. De cette façon nous avons essayé d'éliminer, ou de réduire, les variations climatiques de la surface et l'apport de matériaux exter- 
nes venant des entrées. Durant les prélèvements, on a respecté rigoureusement les normes usuelles d'asepsie. Les sédiments, recueillis avec des cuillères stérilisées, passées sur place à la flamme, ont été versés dans des récipients de verre stérilisés et fermés par des bouchons de coton.

Pour obtenir des échantillons homogènes, on a effectué les prélèvements dans des points différents mais sur une surface limitée de la zone choisie. En éliminant la couche superficielle de $0,5-1 \mathrm{~cm}$, on a effectué les prélèvements jusqu'à une profondeur d'environ $10 \mathrm{~cm}$. Les échantillons prélevés ont été conservés, durant leur transport en laboratoire, dans un récipient thermique portatif, à basse température.

Nous avons effectué cinq prélèvemnets de sédiments à des dates différentes, afin d'observer les éventuelles variations liées au cycle des siaisons. Les dates des prélèvements sont les suivantes: novembre 1972, février, mai, juillet et novembre 1973.

\section{ÉVALUATION DE LA MICROFLORE TOTALE}

Pour l'étude de la microflore totale nous avons utilisé les techniques décrites par Pochon et Tardieux (1962), basées sur la numération indirecte sur milieux solides.

Les suspensions-dilutions, obtenues à partir des échantillons de sédiment, ont été ensemencées sur agar Mycobios*, en boîtes de Petri, à raison de $0,1 \mathrm{ml}$ par boîte. Pour chaque échantillon on a préparé en moyenne vingt boîtes. Sur la base d'un essai préliminaire que nous avions effectué précédemment, les dilutions choisies pour l'ensemencement ont été de $10^{-3}$ pour la grotte de «Punta degli Stretti» et pour la résurgence de «Trevi», et de $10^{-4}$ pour la grotte de la «Mandorla», et cela afin d'obtenir un nombre optimal de colonies par chaque boîte.

Les boîtes ensemencées ont été incubées en thermostat à $28^{\circ} \mathrm{C}$ pendant 7 jours. A la fin de l'incubation on a effectué le comptage en faisant une distinction entre les colonies de Bactéries et d'Actinomycètes et celles de Champignons. Les résultats obtenus ont été exprimés en nombre de microorganismes par gramme de sédiment sec.

\section{ÉVALUATION DES ACTIVITÉS FONCTIONNELLES DU CYCLE DE L'AZOTE}

Les activités fonctionelles du cycle de l'azote que nous avont étudiées sont les suivantes: fixation d'azote aérobie et anaérobie, protéolyse, ammonification, nitrification (bactéries nitreuses et nitriques), dénitrification. Les techniques employées pour les analyses sont celles de Pochon et Tradieux (1962): les suspensions-dilutions des échantillons prélevés ont été ensemencées en milieux

* Biolife (Milan) 
liquides électifs et incubées ensuite en thermostat à $28^{\circ} \mathrm{C}$. On a effectué des lectures périodiques, en évaluant les tubes positifs au moyen de réactifs chimiques ou par observation directe, selon l'activité fonctionnelle. Sur la base des lectures périodiques faites pendant l'incubation on a établi les courbes d'activité biologique, qui expriment l'accroissement des populations microbiennes en fonction du temps et de la dilution.

Enfin, utilisant les tables de McCrady (1918), on a estimé le nombre probable de microorganismes appartenant aux différentes groupements fonctionnels présents dans les sédiments des trois grottes.

Grot tes
A) «Punta degli Stretti»

B) «Mandorla»

C) «Trevi»

XI / 72
II / 73
V / 73
VII / 73
XI / 73

$\mathrm{XI} / 72$

II $/ 73$

$\mathrm{V} / 73$

VII / 73

$\mathrm{XI} / 73$
XI / 72

II / 73

$\mathrm{V} / 73$

VII / 73

XI / 73

\begin{tabular}{|c|c|c} 
Champignons & $\begin{array}{c}\text { Bactéries et } \\
\text { Actinomycètes }\end{array}$ & $\begin{array}{c}\text { Microflore } \\
\text { totale }\end{array}$ \\
\hline & & \\
& & \\
60 & 400 & 460 \\
13 & 124 & 137 \\
5 & 70 & 75 \\
4 & 6519 & 6523 \\
4 & 664 & 668 \\
& & \\
& & \\
1546 & 16511 & 18057 \\
630 & 35631 & 36261 \\
1882 & 62953 & 64835 \\
704 & 65489 & 66193 \\
249 & 27138 & 27387 \\
& & \\
& & \\
6 & 122 & 128 \\
1 & 1032 & 1033 \\
9 & 54 & 63 \\
21 & 479 & 500 \\
5 & 77 & 82 \\
& & \\
& &
\end{tabular}

Tableau 5 - Nombre de colonies $\left(\mathrm{n} \times 10^{3}\right)$ par gramme de sédiment rapporté au poids sec. 


\section{RÉSULTATS \\ STRUCTURE DES MICROBIOCÉNOSES DES SÉDIMENTS}

Les peuplements microbiens des sédiments (tab. 5; fig. 5, 6, 7) sont caractérisés par une abondance notable de microorganismes dans la grotte de la «Mandorla», où la moyenne des prélèvements est d'environ 42 millions de colonies par gramme de sédiment sec*, et par un rare peuplement microbien dans la résurgence de "Trevi» (valeur moyenne 360.000 microorganismes) et dans la grotte de «Punta degli Stretti» (valeur moyenne 1,6 millions de microorganismes). On doit cependant noter que dans cette dernière grotte la microflore était particulièrement abondante en juillet, passant des 75.000 colonies en mai à 6,5 millions. Puisque ce nombre a été confirmé par un prélèvement de contrôle effectué 8 jours plus tard, on en déduit que cela peut s'attribuer à une augmentation exceptionnelle de la matière organique du sédiment, n'ayant constaté aucunne variation significative de la température ni de la teneur en eau.

La comparaison des résultats avec les chiffres reportés par d'autres auteurs (tab. 6) permet de remarquer que la grotte de «Punta degli Stretti» et la résurgence de «Trevi» ont un peuplement microbien correspondant à celui des grottes les plus pauvres, tandis que dans la grotte de la «Mandorla» la microflore correspond à ce qu'on observe seulement dans les grottes de Lascaux (Pochon et coll., 1964) et d'Hautecourt (Gounot, 1967).

Nos résultats confirment substantiellement la pouvreté de la microflore des sédiments de grotte par rapport à celle des sols de surface. A ce sujet il est intéressant de comparer le nombre de microorganismes des sédiments de certaines grottes avec celui des sols qui les recouvrent. Gounot (1967) a trouvé 30 millions de microorganismes dans le limon de la grotte de «Hautecourt» et 250 millions dans le sol au-dessus de la cavité. Toujours Gounot (1968) a compté 18 millions de microorganismes dans le sol au-dessus de la grotte de «Lapphullet» et 2 millions dans le sédiment de la grotte.

En surface les sédiments pauvres en microflore sont ceux des glaciers, où l'on a trouvé de quelques dizaines de milliers à 4 millions de microorganismes (Moiroud, 1970; Gounot, 1970b). Les sols sablonneux de dune à végétation rare et plus encore les sols marécageux sont plus riches: Naviglio et Visonà (1973) y ont observé respectivement de 1 à 8 millions et de 8 à 145 milions de microorganismes. Dans les tourbes acides De Barjac (1956) a trouvé de 12 à 74 millions de germes. Dans des sols de forêt de chêne vert Billès et coll. (1971) on compté de 441 à 950 millions de microorganismes.

Dans la composition de la microflore (tab. 5; fig. 5, 6, 7) on voit une dominante presque absolue des Bactéries et des Actinomycètes par rapport aux Champignons qui, dans trois prélèvement sur les 15 effectués en tout, représentent un pourcentage compris entre le 10 et le $15 \%$, tandis que dans 9 prélè-

\footnotetext{
* Les chiffres successifs sont toujours exprimés en nombre de colonies par gramme de sédiment sec.
} 


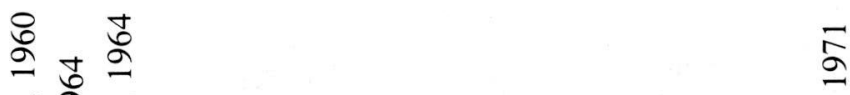

光

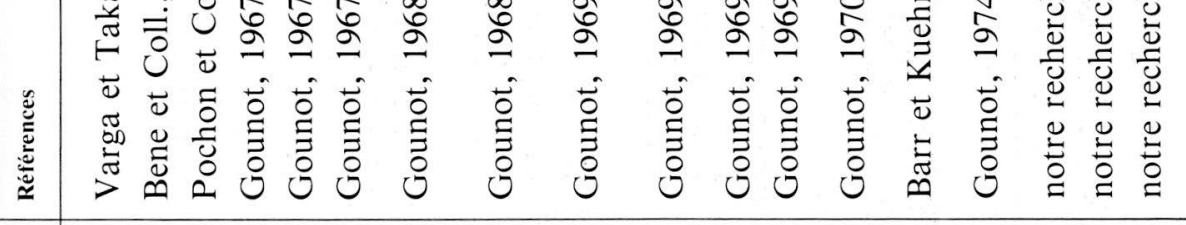

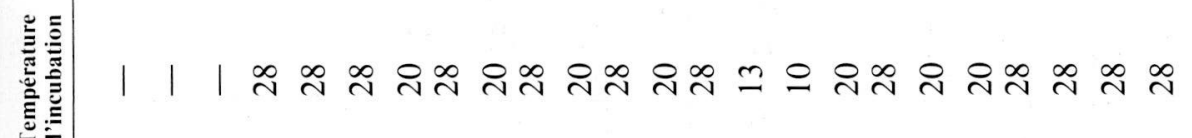

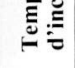

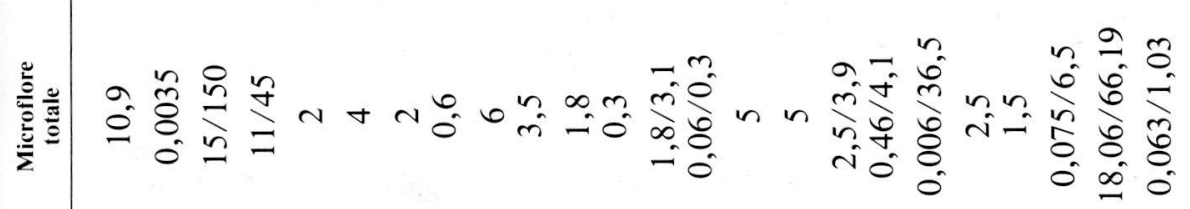

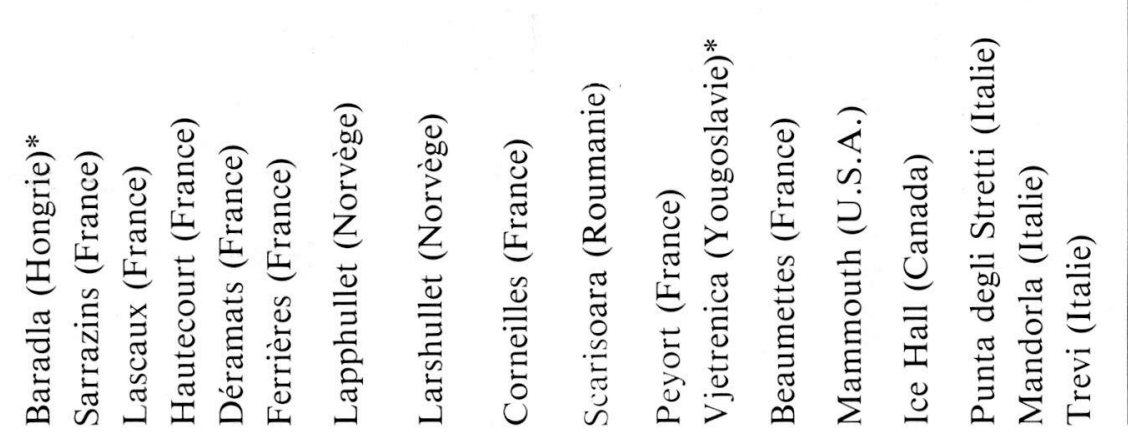

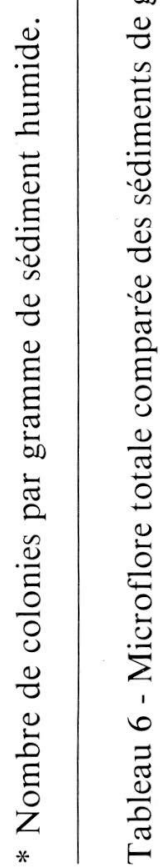




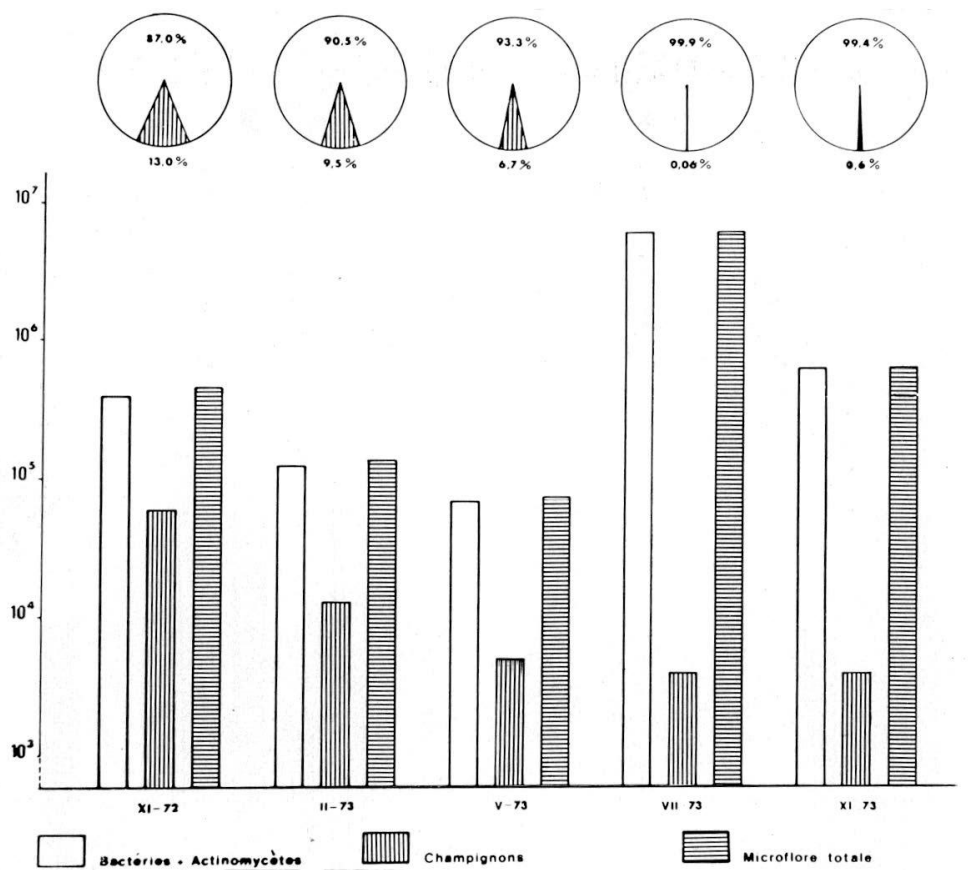

Fig. 5. - Grotte de «Punta degli Stretti». Nombre et pourcentage des microorganismes dans les prélèvements.

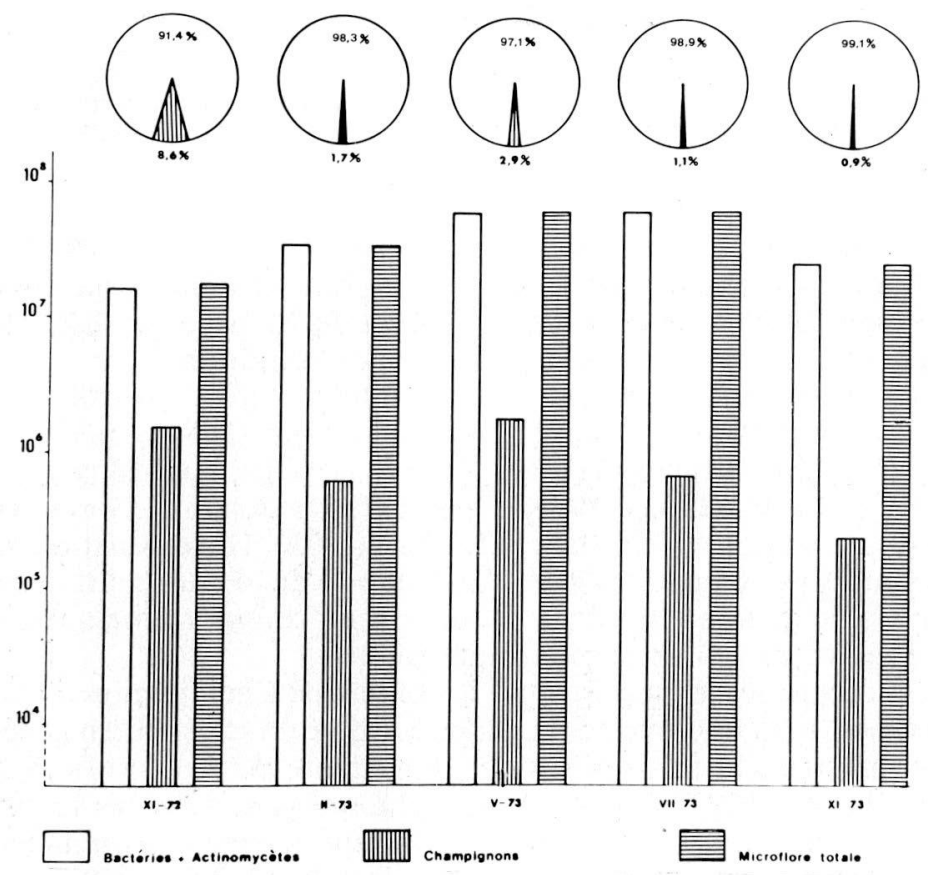

Fig. 6. - Grotte de la «Mandorla». Nombre et pourcentage des microorganismes dans les prélèvements. 

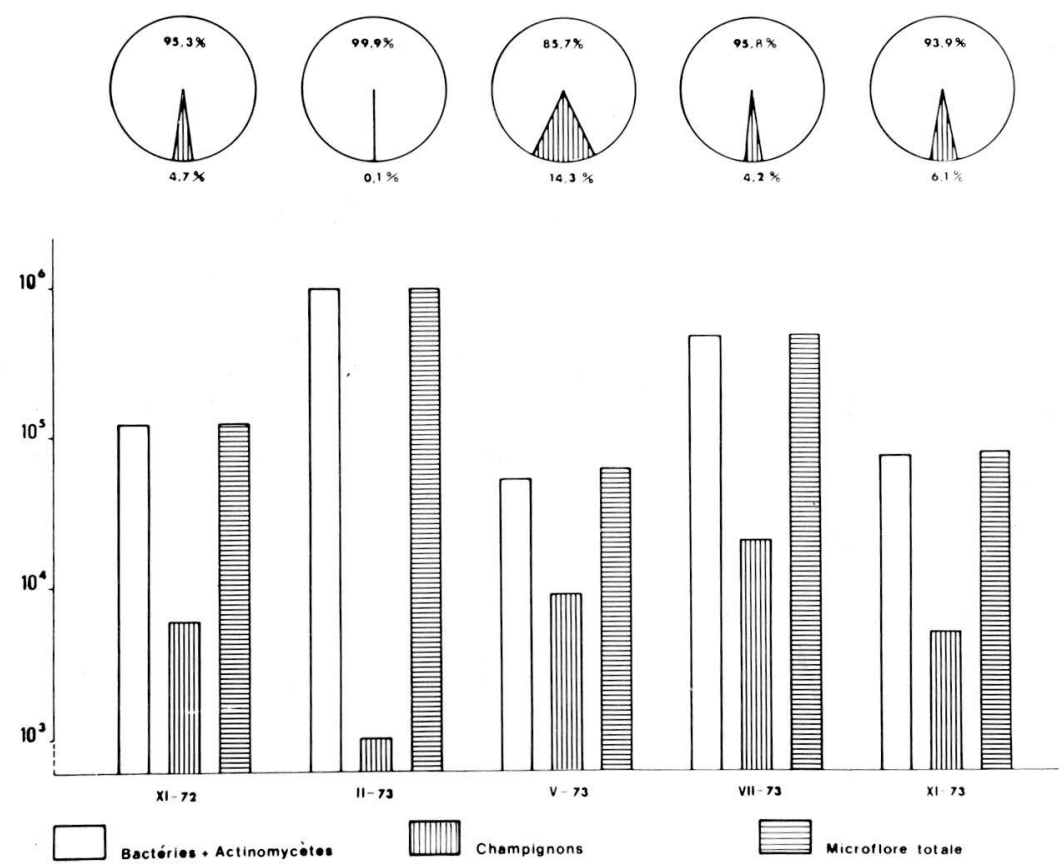

Fig. 7. - Résurgence de «Trevi». Nombre et pourcentuage des microorganismes dans les prélèvements.

vements le nombre des colonies de Champignons n'atteint pas le $5 \%$ de la microflore totale. En particulier les Champignons représentent en moyenne 1\% de la microflore totale dans la grotte de «Punta degli Stretti» et le 2,3\% dans la résurgence de «Trevi» et dans la grotte de la «Mandorla».

Divers auteurs se sont intéressés aux Champignons cavernicoles: Liddo (1951), Barbacovi (1954), Sevazzi (1954), Caumartin (1957b, 1959a, 1961), Bernasconi (1961), Francalancia (1961), Graniti (1962), Luppi-Mosca et Campanino (1962), Martini (1962a, 1963), Orpurt (1964), Anelli et Graniti (1967), Dobat (1970), Gounot (1970b), Barr et Kuehne (1971). Des évaluation quantitatives effectuées par Gounot (1967) dans la grotte de «Hautecourt» ont donné des valeurs de quelques dizaines de milliers de colonies fongiques, correspondant à moins de $1 \%$ de la microflore totale.

Dans les sols de surface le rapport entre le nombre de Champignons et le nombre de Bactéries + Actinomycètes varie avec le type de sol, mais en général les Bactéries constituent la fraction la plus importante de la microflore totale. Naviglio et Visonà (1973), dans des dunes sablonneuses, dans des sol marécageux et des sols alcalins de forêt ont trouvé respectivement les pourcentages suivants de Champignons par rapport à la microflore totale: de 0,75 à $18 \%$, de 0,6 à $4,7 \%$ et de 0,8 à $16 \%$. 
Dans des sols de forêt du Texas, avec un pH compris entre 4,7 et 7,6, BetznerMarrow (1931) a trouvé de 4 à $47 \%$ de Champignons par rapport à la microflore totale.

\section{ACTIVITÉ FONCTIONNELLE DU CYCLE DE L’AZOTE}

Fixation d'azote (tab. 7 et 8 ; fig. 8 et 9).

Le pouvoir de fixation de l'azote moléculaire est faible et est dû, pour la résurgence de «Trevi» et pour la grotte de «Punta degli Stretti», exclusivement à l'activité des fixateurs anaérobies; dans la grotte de la «Mandorla» les microorganismes aérobies, même s'il sont toujours présents, représentent environ $1 / 10$ des anaérobies qui dans cette grotte sont plus nombreux que dans les deux autres.

\begin{tabular}{|c|c|c|c|c|}
\hline \multirow{2}{*}{ Grottes } & \multirow{2}{*}{\multicolumn{2}{|c|}{\begin{tabular}{l}
\multicolumn{2}{c}{ Fixateurs d'azote } \\
aérobies | anaérobies
\end{tabular}}} & \multicolumn{2}{|c|}{ Nitrifiants } \\
\hline & & & nitreux & nitriques \\
\hline \multicolumn{5}{|c|}{ A) «Punta degli Stretti» } \\
\hline $\mathrm{XI} / 72$ & 0 & 6 & 370 & 67 \\
\hline II / 73 & 0 & 6072 & 337 & 33700 \\
\hline $\mathrm{V} / 73$ & 0 & 59 & 328 & 328 \\
\hline VII / 73 & 32 & 319 & 319 & 319 \\
\hline $\mathrm{XI} / 73$ & 0 & 35 & 332 & 35 \\
\hline \multicolumn{5}{|l|}{ B) «Mandorla» } \\
\hline $\mathrm{XI} / 72$ & 87 & 870 & 4 & 4800 \\
\hline II / 73 & 250 & 1184 & 118000 & $6,58 \times 10^{6}$ \\
\hline$V / 73$ & 115 & 2436 & 64000 & 115400 \\
\hline VII / 73 & 35 & 352 & 3500 & 6300 \\
\hline $\mathrm{XI} / 73$ & 11 & 3098 & 3100 & 310 \\
\hline \multicolumn{5}{|l|}{ C) «Trevi» } \\
\hline $\mathrm{XI} / 72$ & 0 & 49 & 489 & 489 \\
\hline II / 73 & 0 & 50 & 496 & 4960 \\
\hline$V / 73$ & 0 & 32 & 71 & 396 \\
\hline VII / 73 & 0 & 0 & 488 & 879 \\
\hline $\mathrm{XI} / 73$ & 0 & 6 & 373 & 374 \\
\hline
\end{tabular}

Tableau 7 - Nombre probable de fixateurs d'azote et de nitrifiants par gramme de sédiment rapporté au poids sec. 

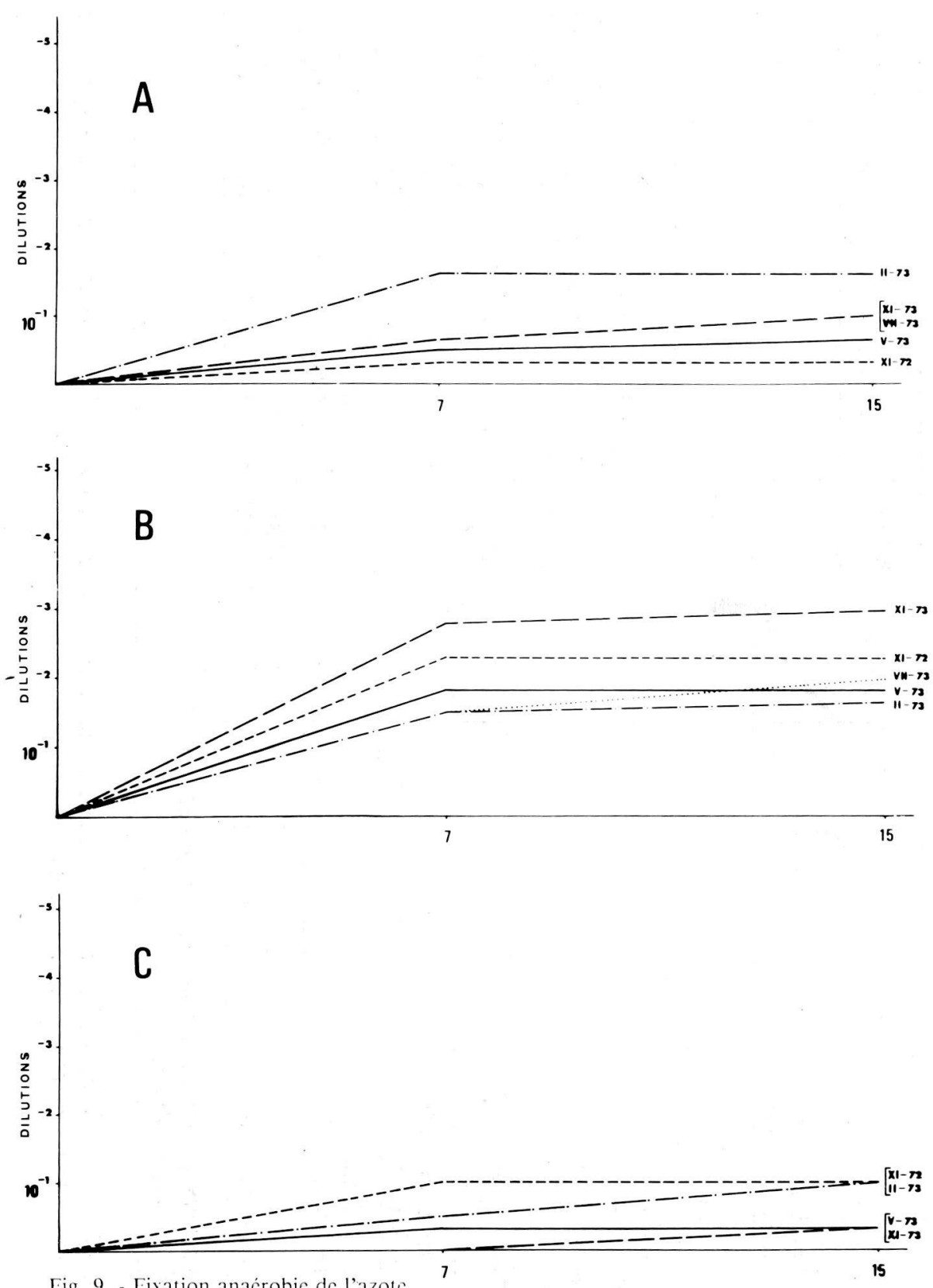

Fig. 9. - Fixation anaćrobic de l'azote.

A) Grotte de «Punta degli Stretti».

B) Grotte de la «Mandorla».

C) Résurgence de «Trevi». 


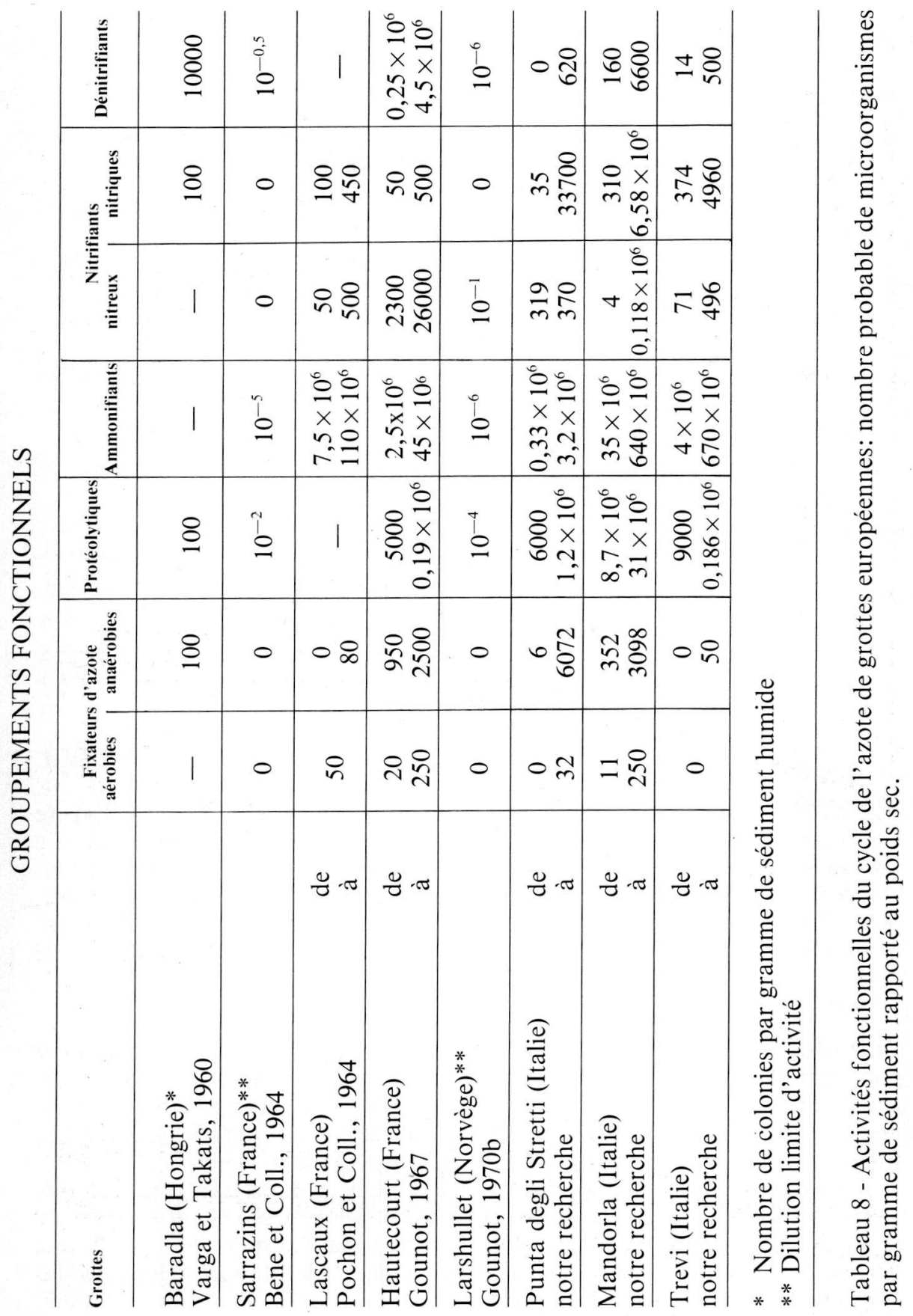




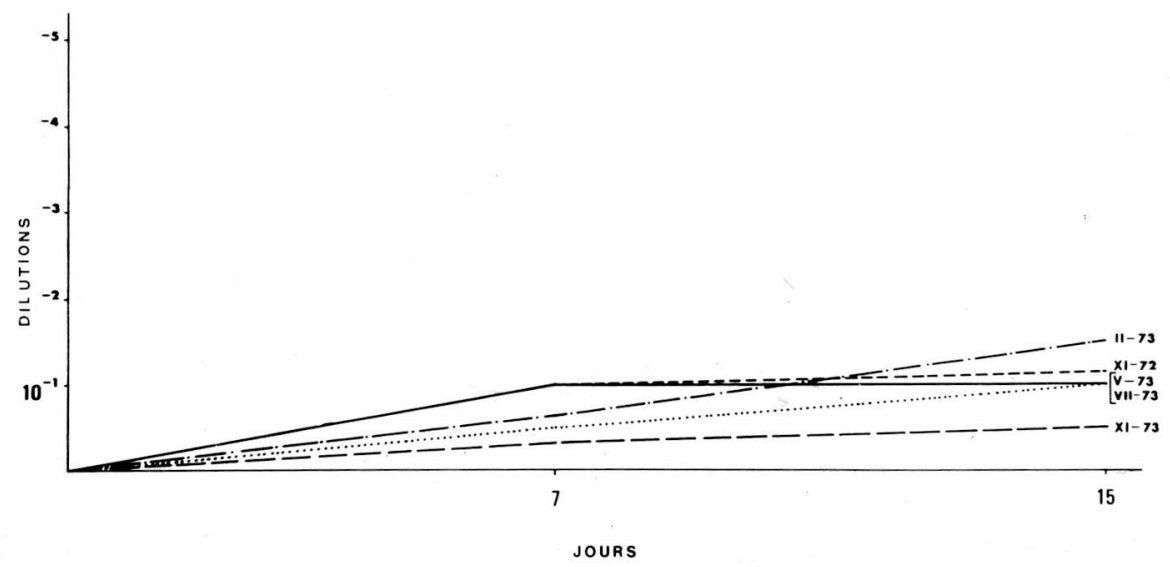

Fig. 8. - Fixation aérobie de l'azote. Grotte de la «Mandorla».

Les chiffres obtenus confirment la pauvreté de la microflore fixatrice d'azote non symbiotique des sédiments des grottes (tab. 8), comme l'ont signalé Mason-Williams et Benson-Evans (1958), Varga et Takats (1960), Martini (1962a), Bene et coll. (1964), Pochon et coll. (1964), Gounot (1967, 1970b). Nos résultats ne diffèrent pas tellement de ceux obtenus pour les sols de surface par Jensen (1950) et par Carini (1963), qui indiquent la pauvreté générale des fixateurs d'azote (Clark, 1967).

Protéolyse (tab. 8; fig. 10). La microflore protéolytique est numériquement plus abondante dans la grotte de la «Mandorla» (des millions de microorganismes) et moins nombreuse à la résurgence de «Trevi» et à la grotte de «Punta degli Stretti» (de milliers à dizaines de milliers de microorganismes), exception faite, dans cette dernière cavité, pour le prélèvement de juillet 1973. Aux différences quantitatives correspond aussi une différence de vitesse de protéolyse. Ce qu'on a observé pour la grotte de la «Mandorla» trouve correspondance dans les chiffres obtenus pour les sols de surface par Carini (1963), tandis que les résultats des deux autres grottes concordent avec les données bibliographiques regardant les sédiments de grotte (Bene et coll., 1964; Gounot, 1967, 1970b) où l'activité protéolytique est inférieure à ce qui est observable en surface. 

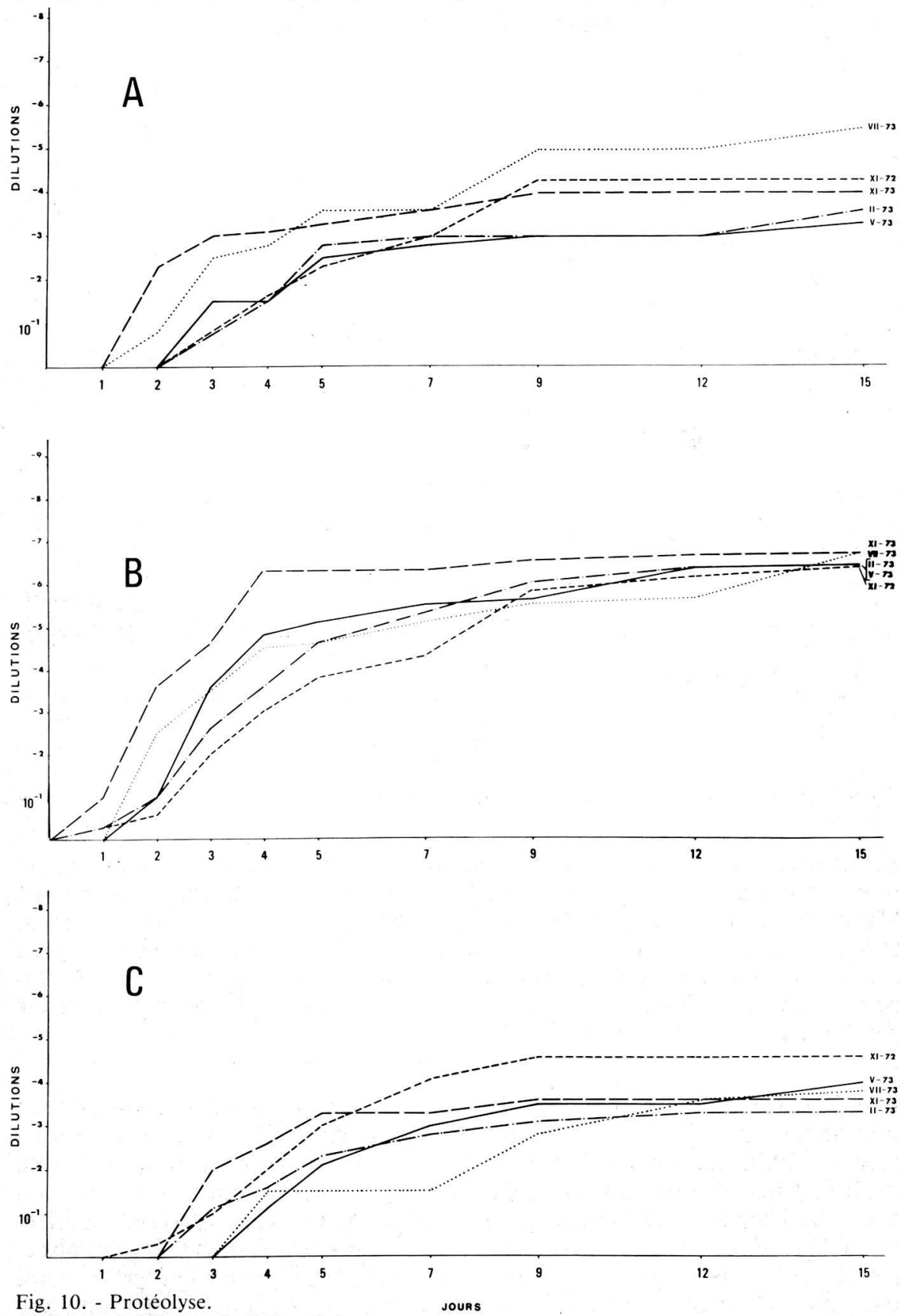
A) Grotte de «Punta degli Stretti».
B) Grotte de la «Mandorla».
C) Résurgence de «Trevi». 

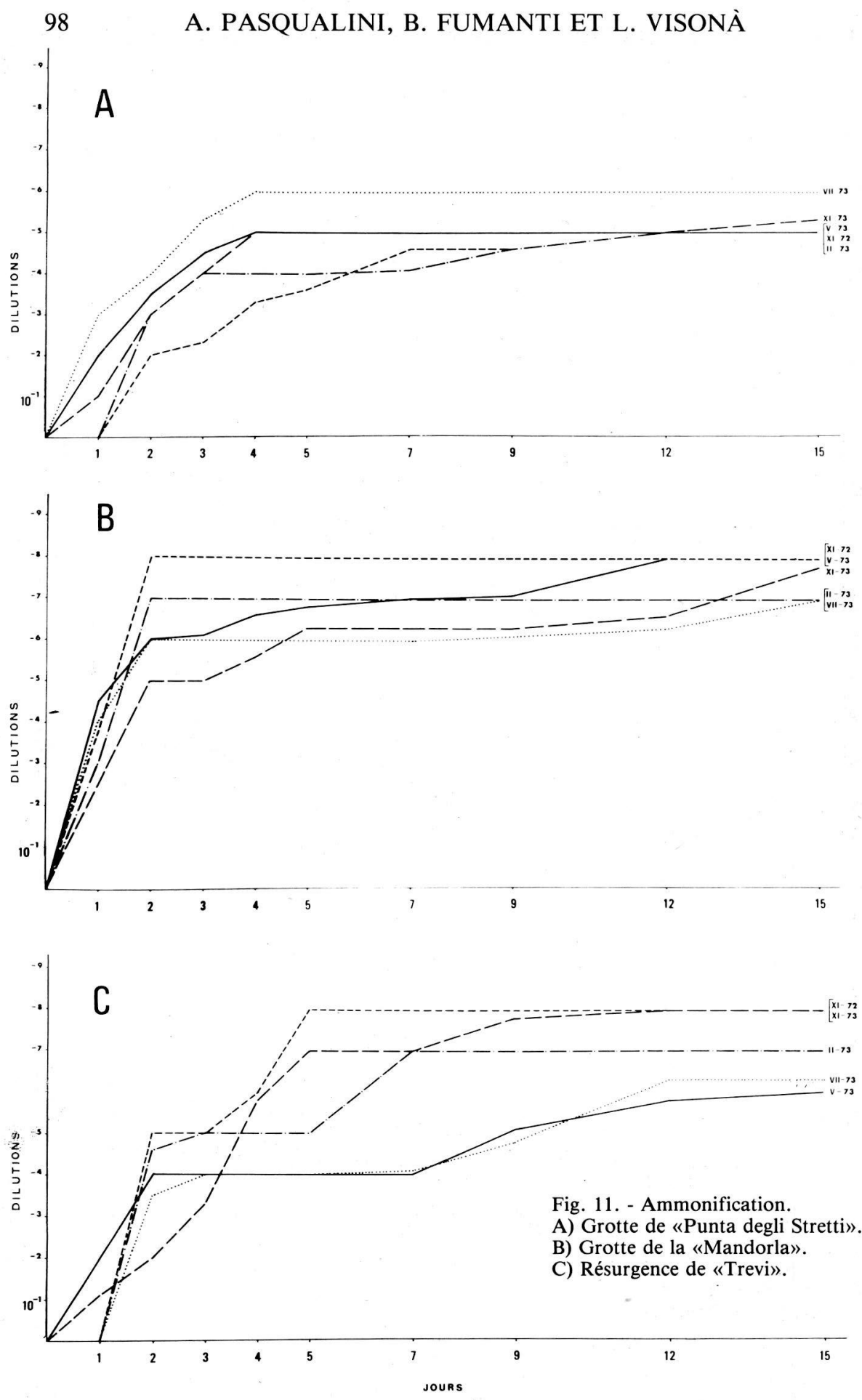

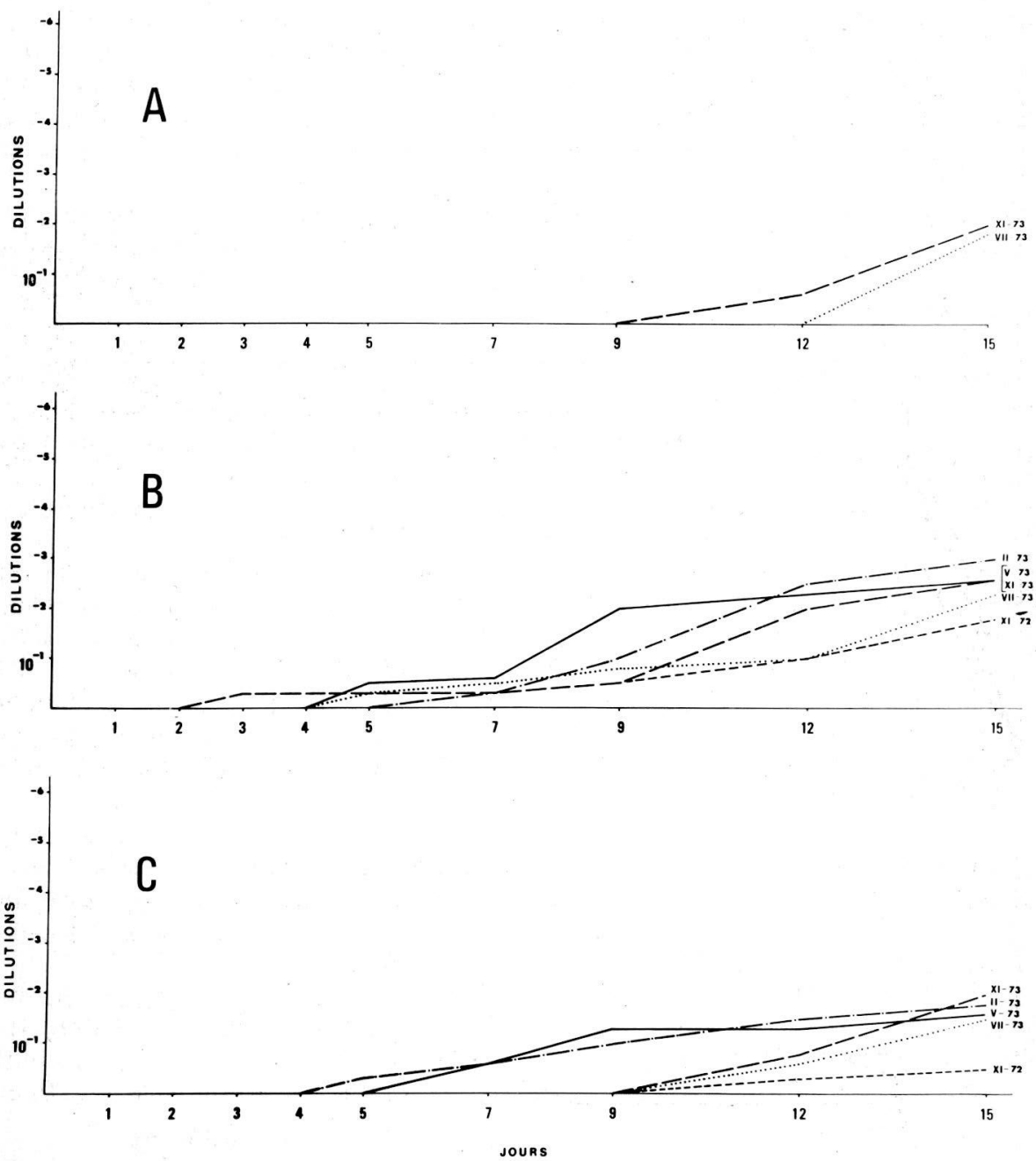

Fig. 12. - Dénitrification.

A) Grotte de «Punta degli Stretti».

B) Grotte de la «Mandorla».

C) Résurgence de «Trevi». 
Ammonification (tab. 8; fig. 11). La microflore ammonifiante est plus nombreuse et plus active que celle des autres groupements fonctionnels. Les différences entre les grottes confirment ce qui a été observé précédemment, c'est-àdire une microflore plus abondante dans la grotte de la «Mandorla» où les microorganismes ammonifiants varient de 35 à 640 millions, se différenciant de celle de la résurgence de "Trevi» seulement pour les valeurs minimum (4 millions). Dans la grotte de «Punta degli Stretti» les ammonifiants sont abondants seulement dans le prélèvement de juillet (3,2 millions). La comparaison entre prélèvements révèle une plus grande variabilité de l'acivité ammonifiante dans la grotte de «Punta degli Stretti» et dans la résurgence de «Trevi» que dans celle de la «Mandorla».

Les chiffres obtenus paraissent plutôt élevés par rapport à ceux signalés par d'autres auteurs pour les sédiments de grotte (tab. 8), tandis qu'ils correspondent, pour la plupart des prélèvements, à ceux des sols de surface (Billès et coll., 1971; Naviglio et Visonà, 1973).

Nitrification. (tab. 7 et 8). Les microorganismes nitreux et nitriques, présents dans tous les échantillons prélevés dans les trois cavités, sont numériquement constants dans la grotte de «Punta degli Stretti» et dans la résurgence de «Trevi», avec des valeurs en général correspondantes à celles citées par d'autres auteurs, tandis qu'à la grotte de la «Mandorla», où ils sont en moyenne plus nombreux, ils présentent une variabilité très marquée.

Des microorganismes nitreux et nitriques ont été trouvés dans l'eau, dans le mondmilch et dans les sédiments de grotte par Mason-Williams et BensonEvans (1958), Fischer (1959b), Varga et Takats (1960), Pochon et coll. (1964), Gounot $(1967,1970 b)$ et Bertouille (1972). Martini (1962a) et Bene et coll. (1964) n'ont pas rélevé, au contraire, une activité nitrifiante dans les sédiments des grottes qu'ils ont étudiées. Même dans les sols de surface la microflore-nitrifiante représente une fraction très basse de la microflore totale (Bardin 1970; Billès et coll., 1971).

Dénitrification (tab. 8; fig. 12). La microflore dénitrifiante, très pauvre numériquement, manifeste aussi une activité spécifique réduite, surtout à la grotte de «Punta degli Stretti» où les microorganismes dénitrifiants sont présents seulement dans deux prélèvements (juillet et novembre 1973).

La présence des dénitrifiants dans le milieu cavernicole a été observée par Fischer (1959a) dans les eaux et par Varga et Takats (1960), Martini (1962a), Bene et coll. (1964), Gounot $(1967,1970 \mathrm{~b})$ dans les sédiments où ils sont beacoup moins nombreux et actifs que dans les sols de surface (Moiroud, 1970; Billès et coll., 1971; Naviglio et Visonà, 1973). 


\section{COMMENTAIRE}

Les résultats obtenus confirment la présence d'une microflore active dans le milieu cavernicole et indiquent que la teneur en matière organique du sédiment est le principal facteur qui en règle l'abondance, même si un rôle complémentaire doit être attribué aux facteurs physiques (température, teneur en eau, $\mathrm{pH}$, granulométrie).

La grotte de la «Mandorla» dont la morphologie permet une pénetration facile des débris organiques de l'extérieur, présente une microflore totale des sédiments de l'ordre de dizaines de millions de microorganismes. Dans la grotte de «Punta degli Stretti» et dans la résurgence de «Trevi», caractérisées au contraire par un meilleur isolement de l'extérieur, les valeurs obtenues sont de l'ordre de dizaines et de centaines de milliers de microorganismes.

La composition de la microflore révèle un pourcentage de Champignons très bas, probablement en relation à l'alcalinité des sédiments $(\mathrm{pH}=7,5-7,6)$ et indique donc combien le rôle des micromycètes est limité dans ce milieu.

Pour ce qui regarde le cycle de l'azote, l'analyse des résultats montre une correspondance significative entre la nature de la matière organique, exprimée par le rapport $\mathrm{C} / \mathrm{N}$, et l'activité des groupements fonctionnels. Dans les sédiments de la résurgence de «Trevi» et surtout de la grotte de la «Mandorla», l'activité intense du cycle de l'azote s'accorde très bien avec le bas rapport $\mathrm{C} / \mathrm{N}$. Au contraire, dans la grotte de «Punta degli Stretti» où le rapport $\mathrm{C} / \mathrm{N}$ est très élevé, les microorganismes des groupements fonctionnels du cycle de l'azote sont moins nombreux et moins actifs.

La présence d'un cycle de l'azote substantiellement complet dans la grotte de «Punta degli Stretti» et dans la résurgence de «Trevi», confirme les observations de Gounot $(1967,1973)$ selon qui des quantités même petites de matière organique peuvent être utilisées avec profit par une microflore hétérotrophe évidemment peu exigeante et capable d'effectuer simultanément plusieurs fonctions.

Le cycle saisonnier ne semble pas influencer de façon significative la quantité et la composition de la microflore des sédiments des trois grottes. L'isolement du milieu cavernicole entraîne en effet, comme on le sait, une remarquable stabilité des conditions climatiques des grottes; il s'ensuit que les variations des paramètres physico-chimiques liées au cycle saisonnier sont très affaiblies. La présence de Bactéries nitrifiantes autotrophes dans les sédiments des grottes étudiées, confirme, comme Gounot l'a déjà observé (1967), la possibilité d'une productivité primaire même dans le milieu cavernicole, où une contribution partielle à cette productivité est donnée aussi par l'activité des Bactéries fixatrices d'azote, hétérotrophes seulement pour le carbone.

Bien que le nombre réduit des Bactéries nitrifiantes et fixatrices d'azote semble indiquer une productivité primaire quantitativement négligeable de ces microorganismes, nous estimons que l'argument mérite des recherches successives pour mieux évaluer la contribution effective des Bactéries chimioautotrophes à la biologie des sédiments dans les grottes. 


\section{RÉSUMÉ}

Étude comparative de la microflore des sédiments de trois grottes italiennes différentes quant à leurs caractéristiques trophiques. Le variations quantitatives et qualitatives de la microflore on été évaluées sur une année, à travers l'étude de la microflore totale et de l'activité des groupements fonctionnels du cycle de l'azote. Les données obtenues ont été comparées avec celles des auteurs précedents.

Les resultats indiquent dans la quantité et dans la nature de la matière organique du sédiment le principal facteur qui règle l'abondance et l'activité de la microflore cavernicole.

La partie expérimentale de cette étude est précédée par un résumé des recherches effectuées jusque'ici sur la microflore cavernicole et par une description des caractéristiques topographiques, géomorphologiques, hydrologiques et trophiques des grottes étudiées.

\section{BIBLIOGRAPHIE}

ANELLI, F., GRANITI, A. 1967 - Aspetti microbiologici nella genesi delle vermicolazioni argillose delle Grotte di Castellana (Murge di Bari). Le Grotte d'Italia, 1:131-139.

ASSOCIAZIONE SPELEOLOGICA ROMANA, 1972-1973. Bollettino. Sous presse.

BARBACOVI, G. 1954 - Funghi cavernicoli, descrizione di una nuova specie di Ascomicete. St. Tr. Sci. Nat., Trento, 31:50-53.

BARDIN, R. 1970 - Contribution à l'étude de la minéralisation de l'azote organique «in situ» et «in vitro» de sols alcalins de Haute-Durance. Rev. Ecol. Biol. Sol., 7:471-492.

BARR, T.C., KUEHNE, A. 1971 - Ecological studies in Mammouth Cave system of Kentucky. II. The ecosystem. Ann. Spél., 26: 47-96.

BASCHIERI-SALVADORI, F. 1952 - Salentinella denticulata, n. sp., Anfipode Gammaride di acque cavernicole del Monte Argentario. Boll. Zool. Napoli, 19:3-7.

BENE, R., GIRARD, T., BALDO, S. 1964 - Etude microbiologique d'un sol de grotte (Grottes des Sarrazins, Lourdes). Bull. Ass. Diplom. Microbiol. Fac. Pharm. Nancy, 96:3-8.

BERNASCONI, R. 1961 - Note sur la flore mycologique cavernicole de Suisse. Schweiz, Zeitschr. Pilsk., 85-92.

BERTOUILLE, H. 1972 - Le mondmilch, repère climatique. Ann. Spél., 27:609-623.

BETZNER-MARROW, M. 1931 - Correlation between plant communities and the reaction and microflora of the soil in South Central Texas. Ecology, 12:497-507.

BILLĖS, G., CORTEZ, J., LOSSAINT, P. 1971 - L'activité biologique des sols dans les écosystèmes méditerranéens. I. Minéralisation du carbone. Rev. Ecol. Biol. Sol., 8:375-395.

BIRSTEIN, J.A., BORUTZKY, E.V. 1950 - Zinz v podziemnych vodlach. Zizn priesnych vod SSSR, 3:683-706.

BOEGLI, A. 1961 - Der Höhlenlehm. Mem. $5^{\circ}$ Rass. Spel. Ital., Como, 21 p.

BOUCART, J., FRANCIS-BOEUF, C. 1942 - La vase. Actual. Scient. Industr., 927. Hérmann éd., Paris, 67p.

CARINI, S., 1963 - «Stanchezza» del medicaio e microflora del terreno. Ann. Microbiol. Enzimol., 13:11-18.

CAUMARTIN, V., 1957a - La microflore des cavernes. Notes Biospéol., 12:59-64.

CAUMARTIN, V. 1957b - Recherches sur une bactérie des argiles de cavernes et des sédiments ferrugineux. C.R. Acad. Sci., Paris, 245:1758-1760.

CAUMARTIN, V. 1959a-Quelques aspects nouveaux de la microflore des cavernes. Ann. Spél.. 14:147-157.

CAUMARTIN, V. 1959b - Morphologie et position systématique du Perabacterium spelei. Bull. Soc. Bot. Nord France, 12:15-17.

CAUMARTIN, V. 1960 - Le rôle de la microflore dans l'évolution des sédiments. Ann. Spél., 15:401.

CAUMARTIN, V. 1961 - La microbiologie souterraine: ses techniques, ses problèmes. Bull. Soc. Bot. Nord France, 14:53-60.

CAUMARTIN, V. 1962 - Besoin en oligoéléments des Ferrobactériales. Propriétés oligodynamiques des argiles de grotte. Spelunca Mém., 2:99-103. 
CAUMARTIN. V. 1963 - Review of the microbiology of underground enviromments. Nat. Spel. Soc. Bull., 25:1-14.

CAUMARTIN, V. 1964a - Le comportement des Moisissures dans le milieu souterrain. C.R. III Congr. Int. Spél., Wien 1961, 3:41-44.

CAUMARTIN, V. 1964b - Essai sur une étude au microscope électronique de la microflore des sédiments argileux de cavernes. Int. J. Spel., 1:1-12.

CAUMARTIN, V. 1964c - Note sur la présence de dérivés de l'acide carbamique dans les grottes, son importance dans la corrosion des roches et la réduction des oxydes métalliques. Spelunca Mém., 4:17-23.

CAUMARTIN, V. 1965 - La corrosion microbienne dans un réseau karstique. «Sous le plancher». Bull. Spél. Club Dijon, 4:34-43.

CAUMARTIN, V., RENAULT, P., 1958 - La corrosion biochimique dans un réseau karstique et la genèse du mondmilch. Notes Biospéo., 13:87-109.

CAVAILLÉ, A. 1960 - Les argiles des grottes. Introduction à l'étude des sédiments souterrains. Ann. Spél. 15:383-400.

CHDOROWSKI, A. 1959 - Les études biospéologiques en Pologne. Biospél. Pol., Speleologia, 1:122-144.

CLARK, F.E. 1967 - in BURGES, A., RAW, F. Soil biology. Academic Press.

CUMIN, G. 1928 - I territori a fenomeni carsici dell'Italia. Le Grotte d'Italia, 2:27-29.

DATTI, S. 1930 - La Grotta di «Punta degli Stretti» (Orbetello). Le Grotte d'Italia, 2:101-106.

DE BARJAC, H. 1956 - Schéma d'analyse des tourbes acides en vue de leur revalorisation. $6^{\circ}$ Congr. Sci. Sol., Paris: 281-286.

DE SIO, F., CHIANCONE, M. 1965 - Elenco catastale delle grotte della Toscana. Atti $6^{\circ}$ Conv. Italia Centro-Meridion., Firenze 1964:206-215.

DOBAT, K., 1970 - Considérations sur la végétation cryptogamique des grottes du Jara-Souabe (Sud-Ouest de l'Allemagne). Ann. Spél., 25:871-907.

DUCCI, A., SEGRE, A.G., 1950 - Cenni sulle brecce ossifere del versante Nord del promontorio Argentario. Boll. Serv. Geol. Italia, 72:65-71.

DUDICH, E. 1930 - Die Nahrungsquellen der Tierwelt in der Aggtelek Tropfsteinhöle. Allat. Közlem., 27:77-85.

DUDICH, E. 1932 - Biologie der Aggtelek Tropfsteihöle «Baradla» in Hungarn. Spel. Monogr., 13:1-246.

DUDICH E., 1933 - Die Klassifikationen der Höhlen auf biologischer Grundlage. Mitt. Höhl. Karstf., 3:35-45.

FISCHER, E. 1959a - Wyniki analizy bakteriologicznej drobnych zbiornikow wodnych groty Zimnej i Krasprowej. Speleologia, 3.

FISCHER, E. 1959b - Bakterie dwoch zbiornikow wodnych jaskin tatrzanskich. Polsk. Arch. Hydrobiol., 6:189-199.

FRANCALANCIA, P.O. 1961 - Flora crittogamica delle Grotte di Castellana. Tesi di laurea in Scienze Naturali; Pont. Ist. Sup. Sci. Lett. S. Chiara, Napoli, 1960-61.

GINET, R. 1960 - Ecologie, éthologie et biologie des Niphargus (Amphipodes Gammaridés hypogées). Ann. Spel., 15:127-376.

GOUNOT, A.M. 1960 - Recherches sur le limon argileux souterraine et sur son rôle nutritif pour les Niphargus (Amphipodes Gammaridès). Ann. Spél., 15:501-526.

GOUNOT, A.M. 1964 - Recherches sur la production de vitamines par la microflore d'un limon argileux souterrain. Spelunca Mém., 4: $178-180$.

GOUNOT, A.M. 1966 - Production de substances organiques de croissance par la microflore des limons argileux souterrains. C.R. Acad. Sci., Paris, 262:524-526.

GOUNOT, A.M. 1967 - La microflore des limon argileux souterrains: son activité productrice dans la biocoenose cavernicole. Ann. Spél., 22:23-143.

GOUNOT, A.M. 1968 - Etude microbiologique de limons de deux grottes arctiques. C.R. Acad. Sci., Paris, 266:1619-1620.

GOUNOT, A.M. 1969a - Activité productrice de la microflore de limons argileux souterrains. Act. $6^{\circ}$ Congr. Int. Spél., 4-5: 103-108

GOUNOT, A.M. 1969b - Etude préliminare du peuplement bactérien du limon de la grotte de Peyort (Ariège). Ann. Spél., 24:565-601.

GOUNOT, A.M. 1969c - Contribution à l'étude des Bactéries des grottes froides. C.R. $5^{\circ}$ Congr. 
Int. Spél., Stuttgart 1969, 4, B23:1-6.

GOUNOT, A.M. 1970a - Action sélective de la température sur le micropeuplement des grottes froides. Spelunca Mém., 7:123-126.

GOUNOT, A.M. 1970b - Quelques observation sur le micropeuplement des limons de grottes arctiques. Bull. Soc. Linn. Lyon, 39:226-236.

GOUNOT, A.M. 1973 - Recherches sur les Bactéries cavernicoles. C.R. $96^{\circ}$ Congr. Nat. Soc. Sav., Toulouse 1971, 3:257-265.

GOUNOT, A.M. 1974 - Analyse microbiologique d'un limon souterrain des Montagnes Rocheuses. Ann. Spél., 29:333-334.

GRANITI, A. 1962 - Scolebasidium anellii n. sp., agente di annerimento superficiale di stalattiti. Giorn. Bot. Ital., 69:360-365.

JENSEN, H.L. 1950 - Int. Congr. Soil. Sci. Amsterdam, 1:165-172.

JUBERTHIE, C., MESTROV, M. 1965 - Sur les Oligochètes terrestres des sédiments argileux des grottes. Ann. Spél., 20:209-236.

LANZA, B. 1954 - Notizie sugli Ortotteri raccolti nelle caverne della Toscana. Eco Spel., Firenze, 1: 7-12.

LIDDO, S. 1951 - Ricerche batteriologiche nell'aria delle grotte di Castellana. Contributo alla studio della microflora cavernicola. Boll. Soc. Ital. Biol. Sper., 27:496-498.

LOTTI, G. 1956 - La determinazione della sostanza organica nel terreno agrario. Ann. Fac. Agr. Univ. Pisa, 17: 113-128.

LOVETT, T. 1949 - Microorganisms in cave. Cave Sci., Bull. Brit. Spel. Ass., 2:51-52.

LUPPI-MOSCA, A.M., CAMPANINO, E. 1962 - Analisi micologiche del terreno di grotte piemontesi. Allionia, 8:27-43.

MAGDEBURG, P. 1933 - Organogene Kalkkonkretionen in Höhlen. Beiträge zur Biologie der in Höhlen verkommenden Algen. Sitzb. Naturf. Gesell. Leipzig, 56-59:14-36.

MAGDEBURG, P. 1935 - Kälsinterbildung durch Höhlenpflanzen. 400 I. Höhlenf. Bayer. Ost., $38-41$.

MARTINI, A. 1962a - Alcune indagini preliminari sulla microflora delle caverne. Rass. Spel. Ital., 14:245-251.

MARTINI, A. 1962b - La microflora della caverna di Monte Cucco. I. I Blastomiceti. II. I degradatori della cellulosa e gli Attinomiceti. III. I fissatori di N, i nitrificanti, gli ammonificanti, i ridutori dei solfati e gli ossidanti dei tiosolfati. Ann. Fac. Agr. Univ. Perugia, 17.

MARTINI, A. $1962 \mathrm{c}$ - La flora microbica nei terricci di riempimento delle caverne. Atti $4^{\circ} \mathrm{Conv}$. Spel. Italia Centr., Perugia.

MARTINI, A. 1963 - Yeast in cavern environments. Arch. Mikrobiol. 45:111-114.

MASON-WILLIAMS, A. 1959 - The formation and deposition of mondmilk. Cave Res. Group Great Brit. Trans., 5:133-138.

MASON-WILLIAMS, A. 1965 - Comments on the bacterial population of small pools in caves. C.R. $4^{\circ}$ Congr. Int. Spél., Ljubljana.

MANSON-WILLIAMS, A., BENSON-EVANS, K. 1958 - A preliminary investigation into the bacterial and botanical flora of caves in South Wales. Cave Res. Group Great Brit., 8:70p.

MAXIA, C. 1956 - Bibliografia geologica d'Italia. Lazio. Ist. Geol. Paleontol. Univ. Roma.

McCRADY, M.H. 1918 - Tables for rapid interpretation of fermentation tube results. Can. Pub. Health J., 9:201.

MOIROUD, A. 1970 - Etude microbiologique des limons de deux glaciers alpins. I. Activité biologique à basse température. Rev. Ecol. Biol. Sol., 7:457-464.

MOLNAR, M. 1961 - Beiträge zur Kenntnis der Mikrobiologie der Aggtelek Tropfsteinhohle «Baradla» Ann. Univ. Sci. Budapest, Biol., 4:131-139.

NAVIGLIO, L., VISONȦ, L. 1973 - Ricerche preliminari sulla microflora e sulla attività dei gruppi funzionali del ciclo dell'azoto nel rifugio faunistico di Bolgheri (Livorno). Ann. Bot. (Roma). 32:129-154.

ORPURT, P.A. 1964 - The microfungal flora of bate cave soils fron Elenthera Island, the Bahamas. Canad. J. Bot., 42:1629-1633.

PERRIER, J. 1970 - Influence du facteur température sur les groupements fonctionnels d'un sol superficiel et du limon souterrain correspondant. C.R. $9^{\circ}$ Cong. Nat. Spél., Dijon 1970, Spelunca Mém., 7:141-144. 
PERRIER, J. 1971 - Répartition des bactéries dans les limons argileaux souterrains en fontion des facteurs écologiques. C.R. $96^{\circ}$ Congr. Nat. Soc. Sav., Toulouse 1971, Sciences, 3:211-225.

POCHON, J., DE BARJAC, H. 1958 - Traité de microbiologie des sols. Applications agronomiques. Dunod éd., Paris, 685p.

POCHON, J., TARDIEUX, P. 1962. Techniques d'analyse en microbiologie du sol. La Tourelle éd., St-Mandé, $111 \mathrm{p}$.

POCHON, J., CHALVIGNAC, M.A., KRUMBEIN, W. 1964 - Recherches microbiologiques sur le mondmilch. C.R. Acad. Sci., Paris, 258:5113-5115.

POHL, E.R., WHITE, W.B. 1965 - Sulfate minerals: their origin in the central Kentucky karst. Amer. Min., 50:1461-1465.

POLITI, I. 1939-1940 - Sui fenomeni di annerimento delle formazioni calcitiche e calcaree nelle Grotte di Postumia. Le Grotte d'Italia, 4:18-22.

RICCARDI, R. 1927 - Su alcune cavità sotterranee presso S. Filippo (Sabina). Boll. Reale. Soc. Geogr. Ital., 4:146-153.

SCHREIBER, G, 1929 - Il contenuto di sostanza organica nel fango delle Grotte di Postumia. Atti Accad. Sci. Ven. Trent. Istr., 20:51-53.

SCHREIBER, G. 1930 - L'azoto alimentare degli animali cavernicoli di Postumia. Considerazioni sul ciclo dell'azoto. Arch. Zool. Ital., 16:650-653.

SBORDONI, V., ARGANO, R., VOMERO, V., ZULLINI, A. 1977 - Ricerche sulla fauna cavernicola del Chiapas (Messico) e delle regioni limitrofe: grotte esplorate nel 1973 e nel 1975. Criteri per una classificazione biospeleologica delle grotte. In «Subterranean fauna of Mexico», parte III; Quad. Accad. Naz. Lincei, n. 171:5-74.

SEGRE, A.G. 1948 - I fenomeni carsici e la speleologia del Lazio. Ist. Geogr. Univ. Roma, Serie A, n. 7.

SERVAZZI, O. 1954 - Su di un interessante micromicete cavernicolo (Parenzaniae sybillae n.gen., n.sp.). Boll. Soc. Nat. Napoli, 63, suppl. 20:1-7.

STANGENBERG, M. 1949 - Nitrogen and carbon in the bottom-deposits of lakes and in the soils under carps-ponds. Verh. Int. Ver. Limnol., 10:422-437.

STELLA, E. 1951a - Monodella argentarii n.sp. di Thermosbenacea (Crustacea, Peracarida) limnotroglobio di Monte Argentario. Arch. Zool. Ital., Napoli, 36:1-15.

STELLA, E. 1951b - Notizie biologiche su Monodella argentarii Stella, Termosbenaceo di una grotta di Monte Argentario. Bol. Zool., Napoli, 18:227-233.

STELLA, E. 1953 - Sur Monodella argentarii Stella, espèce de Crustacé Thermosbenacé des eaux d'une grotte de l'Italie centrale. Hydrobiologia, 5.

STELLA, E. 1955 - Behaviour and developpement of Monodella argentarii Stella, a Thermosbenacen from Italian cave. Verh. Int. Ver. Limnol., 12:464-466.

STELLA, E. 1959 - Ulteriori osservazioni sulla riproduzione e lo sviluppo di Monodella argentarii. Riv. Biol. Ital., 51.

STELLA, E., BASCHIERI-SALVADORI, F. 1953 - La fauna acquatica della Grotta di Punta degli Stretti. Arch. Zool. Ital., 38:441-483.

Touring Club Italiano, 1957 - Conosci l'Italia. Vol. I, l’Italia fisica. Ed. T.C.I., Milano.

VARGA, I., TAKATS, T. 1960 - Mikrobiologische Untersuchungen der Schlammes eines wasserlosen Teiches der Aggtelek BaradlaHöhle. Acta Zóol. Acad. Sci. Hung., 6:429-437. 